\title{
SECONDARY HOMOTOPY GROUPS
}

\author{
HANS-JOACHIM BAUES AND FERNANDO MURO
}

\begin{abstract}
Secondary homotopy groups supplement the structure of classical homotopy groups. They yield a track functor on the track category of pointed spaces compatible with fiber sequences, suspensions and loop spaces. They also yield algebraic models of $(n-1)$-connected $(n+1)$-types for $n \geq 0$.
\end{abstract}

\section{INTRODUCTION}

The computation of homotopy groups of spheres in low degrees in Tod62 uses heavily secondary operations termed Toda brackets. Such bracket operations are defined by pasting tracks where a track is a homotopy class of homotopies. Since Toda brackets play a crucial role in homotopy theory it seems feasible to investigate the algebraic nature of tracks. Therefore we shift focus from homotopy groups $\pi_{n} X$ to secondary homotopy groups

$$
\pi_{n, *} X=\left(\pi_{n, 1} X \stackrel{\partial}{\longrightarrow} \pi_{n, 0} X\right)
$$

defined in this paper. Here $\partial$ is a homomorphism of groups with Coker $\partial=\pi_{n} X$ and $\operatorname{Ker} \partial=\pi_{n+1} X$.

The groups $\pi_{n, 0} X$ and $\pi_{n, 1} X$ are defined directly by use of continuous maps $f: S^{n} \rightarrow X$ and tracks of such maps to the trivial map, so that $\pi_{n, *} X$ is actually a functor in $X$. For $n \geq 2$ the definition involves the new concept of Hopf invariant for tracks.

We show that the homomorphism $\partial$ has additional algebraic structure, namely $\pi_{1, *} X$ is a crossed module, $\pi_{2, *} X$ is a reduced quadratic module and $\pi_{n, *} X, n \geq 3$, is a stable quadratic module.

Crossed modules were introduced by J. H. C. Whitehead in Whi49 and, in fact, for a $C W$-complex $X$ our secondary homotopy group $\pi_{1, *} X$ is weakly equivalent to the crossed module

$$
\pi_{2}\left(X, X^{1}\right) \longrightarrow \pi_{1} X^{1}
$$

studied by Whi49]. Similarly $\pi_{n, *} X$ for $n \geq 2$ is weakly equivalent to the quadratic modules obtained in Bau91 in terms of the cell structure of $X$ which can also be derived from the Kan loop simplicial group associated to $X$, see for example Con84] and BCC93.

The topological and functorial definition of secondary homotopy groups $\pi_{n, *} X$ is crucial to understand new properties of these concepts in the literature. For

1991 Mathematics Subject Classification. 18D05, 55Q25, 55S45.

Key words and phrases. secondary homotopy groups, track category, crossed module, reduced (stable) quadratic module, Hopf invariant of tracks.

The second author was partially supported by the project MTM2004-01865 and the MEC postdoctoral fellowship EX2004-0616. 
example, we are able to determine the algebraic properties of the loop and suspension operators on secondary homotopy groups. As main new results, we describe the fiber sequence for secondary homotopy groups, and we show that secondary homotopy groups form a track functor on the track category of pointed spaces.

In a sequel of this paper we determine the algebraic nature of smash product operations on secondary homotopy groups. For the (stable) secondary homotopy groups of spectra this leads to an algebraic invariant approximating the smash product of spectra.

The computation of the algebra of secondary cohomology operations in Bau shows examples where secondary homotopy groups can be algebraically determined successfully. It is the aim of the authors to generalize the theory of [Bau], concerning the Eilenberg-MacLane spectrum, for general spectra.

Moreover, we will discuss in a sequel of this paper generalized Whitehead products for secondary homotopy groups. In fact, J. H. C. Whitehead introduced in Whi41 Whitehead products as an additional algebraic structure of homotopy groups. We may consider the secondary homotopy groups together with their algebraic properties also as such an enriching structure.

The required "quadratic algebra" associated to properties of secondary homotopy groups is studied in BJP05.

\section{TRACKS BETWEEN MAPS}

We consider the category Top* of compactly generated pointed spaces $X=$ $(X, *)$ and pointed maps $f: X \rightarrow Y$. For any (unpointed) space $X$ we define $X_{+}=X \sqcup\{*\}$ as the same space with an outer base-point $*$. The smash product of two pointed spaces is defined by

$$
X \wedge Y=(X \times Y) /(X \times * \cup * \times Y)
$$

It is strictly associative.

Homotopies $I X \rightarrow Y$ are defined by using the reduced cylinder $I X=I_{+} \wedge X$, where $I=[0,1]$ is the unit interval, with structure maps

$$
X \vee X \stackrel{i}{\longrightarrow} I X \stackrel{p}{\longrightarrow} X
$$

Here $\vee$ is the symbol for the coproduct, $i$ is the inclusion of the boundary and $p$ is the projection. Given two maps $f, g: X \rightarrow Y$ a track $H: f \Rightarrow g$ is a homotopy class of homotopies $I X \rightarrow Y$, from $f$ to $g$, relative to the boundary. By abuse of language we denote a homotopy and the represented track by the same symbol. In diagrams tracks will be denoted as follows.

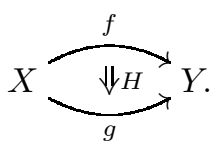


The trivial track 0 : $f \Rightarrow f$ is represented by $f p: I X \rightarrow Y$ and the inverse of a track $H: f \Rightarrow g$ is $H^{\boxminus}: g \Rightarrow f$. The vertical composition of tracks

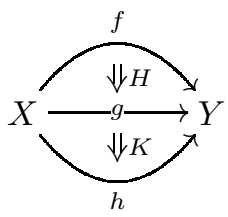

is defined by pasting homotopies representing $H$ and $K$ and is denoted by

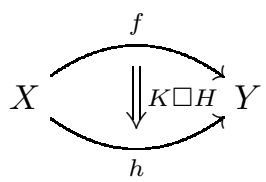

One can also compose horizontally a track as in diagram (1.2) with maps $k: W \rightarrow X$ and $l: Y \rightarrow Z$ to obtain tracks

$$
H k: f k \Rightarrow g k \text { and } l H: l f \Rightarrow l g
$$

in the obvious way. If we have a diagram like

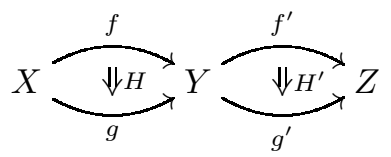

the equality

$$
\left(g^{\prime} H\right) \square\left(H^{\prime} f\right)=\left(H^{\prime} g\right) \square\left(f^{\prime} H\right)
$$

holds and this element is the horizontal composition of $H$ and $H^{\prime}$ denoted by juxtaposition

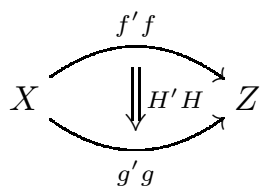

Tracks endow Top* with the structure of a groupoid-enriched category, which we call a track category.

The track category Top* has a strict zero object, the one-point space *. In particular zero maps are defined. Such a track category has the crucial property that any track composed with a zero map becomes automatically a trivial track.

Maps from a coproduct $X \vee Y$ in Top ${ }^{*}$ are given by pairs of maps $\left(f_{1}, f_{2}\right): X \vee$ $Y \rightarrow Z$. Similarly a track $H:\left(f_{1}, f_{2}\right) \Rightarrow\left(g_{1}, g_{2}\right)$ between maps $\left(f_{1}, f_{2}\right),\left(g_{1}, g_{2}\right): X \vee$ $Y \rightarrow Z$ is given by a pair of tracks $H=\left(H_{1}, H_{2}\right)$ with $H_{i}: f_{i} \Rightarrow g_{i}(i=1,2)$.

The suspension $\Sigma X$ is the quotient space $I X /(X \vee X)=S^{1} \wedge X$. We will use the identifications

$$
\Sigma(X \vee Y)=\Sigma X \vee \Sigma Y, \quad \Sigma^{n} S^{0}=S^{n}, \quad n \geq 0 .
$$

For the definition of homotopy groups we choose a particular co-H-group structure on $S^{1}$ given by maps $\mu: S^{1} \rightarrow S^{1} \vee S^{1}$ and $\nu: S^{1} \rightarrow S^{1}$ satisfying the usual properties. We use explicitly these maps in many constructions throughout this 
paper, however these constructions do not depend on this choice since the maps $\mu$ and $\nu$ are unique up to a canonical track.

The loop space functor $\Omega$ is the right-adjoint of the suspension $\Sigma$. The adjoint of a map $f: \Sigma X \rightarrow Y$ is denoted by $a d(f): X \rightarrow \Omega Y$. The adjoint of the identity map 1: $\Sigma X \rightarrow \Sigma X$ is a natural inclusion

$$
\operatorname{ad}(1): X \hookrightarrow \Omega \Sigma X .
$$

As a pointed set the $n$-fold loop space $\Omega^{n} X$ is the set of pointed maps $S^{n} \rightarrow X$ and the base-point corresponds to the trivial map. By using the interchange homeomorphism of the smash product we see that suspensions and cylinders commute up to natural isomorphism in Top*,$I \Sigma X \cong \Sigma I X$. However one has to be careful with signs because the interchange of factors in $S^{1} \wedge S^{1}=S^{2}$ induces -1 on the homotopy group $\pi_{2}$.

\section{Groups of NiLPotency DEGREe 2}

Consider the forgetful functor from groups to pointed sets $\mathbf{G r} \longrightarrow$ Set $^{*}$. This functor has a left adjoint

$$
\langle\cdot\rangle: \text { Set }^{*} \longrightarrow \mathbf{G r}: A \mapsto\langle A\rangle .
$$

Here $\langle A\rangle$ is the quotient of the free group with basis $A$ by the normal subgroup generated by the base-point $* \in A$. This group is isomorphic to the free group with basis $A-\{*\}$. We denote $\vee_{A} S^{1}=\Sigma A$. As usual we identify the fundamental group of $\vee_{A} S^{1}$ with a free group, i. e. $\pi_{1}\left(\vee_{A} S^{1}\right)=\langle A\rangle$. The free group of nilpotency class 2 (free nil-group for short), generated by the pointed set $A$, is the quotient

$$
\langle A\rangle_{n i l}=\frac{\langle A\rangle}{\Gamma_{3}\langle A\rangle}
$$

where $\Gamma_{3}\langle A\rangle$ is the $3^{\text {rd }}$ term of the lower central series of $\langle A\rangle$, i. e. the subgroup generated by triple commutators $[x,[y, z]](x, y, z \in\langle A\rangle)$. In this paper we always write group laws additively, even for non-abelian groups, so that the commutator is $[x, y]=-x-y+x+y$. The free abelian group $\mathbb{Z}[A]$ on a pointed set $A$ is the abelianization of $\langle A\rangle$ and of $\langle A\rangle_{\text {nil }}$.

If $\mathbf{g r}$, nil and $\mathbf{a b}$ are the categories of free groups, free nil-groups and free abelian groups, respectively, then there are obvious nilization and abelianization functors
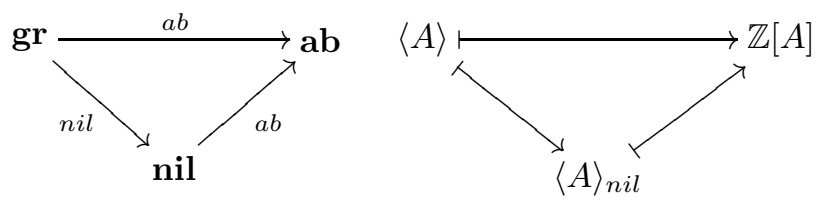

Let $\langle A\rangle_{\text {nil }} \rightarrow \mathbb{Z}[A]$ be the natural projection carrying $x$ to $\{x\}$. Since the commutator bracket in $\langle A\rangle_{\text {nil }}$ is bilinear the homomorphism

$$
\partial: \otimes^{2} \mathbb{Z}[A] \rightarrow\langle A\rangle_{n i l}, \quad \partial(\{x\} \otimes\{y\})=[x, y],
$$

is well defined. Here the tensor square of an abelian group $A$ is denoted by $\otimes^{2} A=$ $A \otimes A$. Let $T: A \otimes B \rightarrow B \otimes A$ be the interchange isomorphism $T(a \otimes b)=b \otimes a$. The reduced tensor square is the following cokernel

$$
\otimes^{2} A \stackrel{1+T}{\longrightarrow} \otimes^{2} A \stackrel{\bar{\sigma}}{\rightarrow} \hat{\otimes}^{2} A .
$$


We denote $\bar{\sigma}(a \otimes b)=a \hat{\otimes} b$. We define the functor $\otimes_{n}^{2}$ as

$$
\otimes_{n}^{2}= \begin{cases}\otimes^{2}, & \text { if } n=2 ; \\ \hat{\otimes}^{2}, & \text { if } n \geq 3 .\end{cases}
$$

Here we write $a \otimes b \in \otimes_{n}^{2} A$ with $a \otimes b=a \hat{\otimes} b$ for $n \geq 3$. Moreover, $\Gamma_{n}$ is the functor

$$
\Gamma_{n}= \begin{cases}\Gamma, & \text { if } n=2 ; \\ -\otimes \mathbb{Z} / 2, & \text { if } n \geq 3 ;\end{cases}
$$

where $-\otimes \mathbb{Z} / 2$ is the ordinary tensor product of abelian groups and $\Gamma$ is Whitehead's universal quadratic functor, see Whi50. There is a natural exact sequence

$$
\Gamma_{n} \mathbb{Z}[A] \hookrightarrow \otimes_{n}^{2} \mathbb{Z}[A] \stackrel{\partial}{\longrightarrow}\langle A\rangle_{n i l} \rightarrow \mathbb{Z}[A] .
$$

Here the first arrow is induced by the function sending $x \in \mathbb{Z}[A]$ to $x \otimes x \in$ $\otimes_{n}^{2} \mathbb{Z}[A]$, see for example Bau91. Moreover, these exact sequences fit into a natural commutative diagram

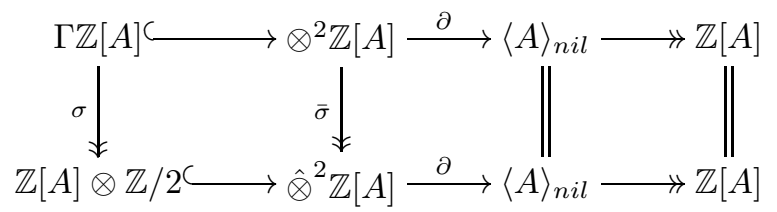

\section{NiL-TRACKS AND HOPF INVARIANTS OF TRACKS}

We now introduce nil-tracks and Hopf invariants of tracks which are needed in the definition of secondary homotopy groups in the next section.

Definition 3.1. Let $f, g$ be maps $f, g: S^{1} \rightarrow \vee_{A} S^{1}$ where $A$ is a discrete pointed set, and let

$$
\Sigma^{n-1} f, \Sigma^{n-1} g: S^{n} \rightarrow \vee_{A} S^{n}
$$

be their $(n-1)$-fold suspensions, $n \geq 1$. A track $H: \Sigma^{n-1} f \Rightarrow \Sigma^{n-1} g$, represented by a homotopy $H: I S^{n} \rightarrow \vee_{A} S^{n}$, is said to be a nil-track if the adjoint

$$
\operatorname{ad}(H): I S^{1} \longrightarrow \Omega^{n-1} \vee_{A} S^{n}
$$

induces a trivial homomorphism

$$
0=H_{2} a d(H): H_{2}\left(I S^{1}, S^{1} \vee S^{1}\right) \longrightarrow H_{2}\left(\Omega^{n-1} \vee_{A} S^{n}, \vee_{A} S^{1}\right) .
$$

The adjoint of $H$ sends the boundary of the cylinder $I S^{1}$ into $\vee_{A} S^{1}$ since $H$ restricted to the boundary is an $(n-1)$-fold suspension. Of course for $n=1$ all tracks $H$ above are nil-tracks since $H_{2} a d(H)$ maps to the trivial group.

Let $f, g$ be now maps between wedges of 1-spheres $f, g: \vee_{B} S^{1} \rightarrow \vee_{A} S^{1}$, and let $\Sigma^{n-1} f, \Sigma^{n-1} g$ be their $(n-1)$-fold suspensions. A track $H: f \Rightarrow g$ is a nil-track if all restricted tracks $H i_{b}$ are nil-tracks where $i_{b}: S^{n} \rightarrow \vee_{B} S^{n}$ is the inclusion given by $b \in B-\{*\}$.

The homology groups involved in the definition of nil-tracks are computable. Indeed,

$$
H_{2}\left(I S^{1}, S^{1} \vee S^{1}\right) \cong H_{2}\left(\Sigma S^{1}\right)=H_{2} S^{2}=\mathbb{Z} .
$$


Moreover,

$$
H_{2}\left(\Omega^{n-1} \vee_{A} S^{n}\right) \stackrel{\cong}{\longrightarrow} H_{2}\left(\Omega^{n-1} \vee_{A} S^{n}, \vee_{A} S^{1}\right)
$$

is an isomorphism, and the Pontrjagin product

$$
\otimes^{2} \mathbb{Z}[A]=H_{1}\left(\Omega^{n-1} \vee_{A} S^{n}\right) \otimes H_{1}\left(\Omega^{n-1} \vee_{A} S^{n}\right) \longrightarrow H_{2}\left(\Omega^{n-1} \vee_{A} S^{n}\right)
$$

is an isomorphism for $n=2$ and induces an isomorphism for $n \geq 2$

$$
\otimes_{n}^{2} \mathbb{Z}[A] \cong H_{2}\left(\Omega^{n-1} \vee_{A} S^{n}\right),
$$

compare notation in (2.2.).

Definition 3.3. Let $n \geq 2$. Given a track $H: \Sigma^{n-1} f \Rightarrow \Sigma^{n-1} g$ for maps $f, g: S^{1} \rightarrow$ $\vee_{A} S^{1}$ the Hopf invariant of $H$ is defined as

$$
\operatorname{Hopf}(H)=\left(H_{2} a d(H)\right)(1) \in \otimes_{n}^{2} \mathbb{Z}[A],
$$

where we apply the homology functor $H_{2}$ as in Definition 3.1 In particular, $H$ is a nil-track if and only if $\operatorname{Hopf}(H)=0$. More generally, if $H: \Sigma^{n-1} f \Rightarrow \Sigma^{n-1} g$ is a track for maps $f, g: \vee_{B} S^{1} \rightarrow \vee_{A} S^{1}$ the Hopf invariant of $H$ is the homomorphism

$$
H o p f(H): \mathbb{Z}[B] \longrightarrow \otimes_{n}^{2} \mathbb{Z}[A]
$$

defined by $\operatorname{Hopf}(H)(b)=\operatorname{Hopf}\left(H i_{b}\right)$, where $i_{b}: S^{1} \subset \vee_{B} S^{1}$ is the inclusion of the factor corresponding to $b \in B-\{*\}$. Such a track $H$ is a nil-track if and only if $\operatorname{Hopf}(H)=0$.

In case $n=1$ then $\operatorname{Hopf}(H)=0$ for any track $H$ as above.

Remark 3.4. Any element $x \in \pi_{3} \vee_{A} S^{2}$ determines a track $x: 0 \Rightarrow 0$ for the trivial map 0: $S^{2} \rightarrow \vee_{A} S^{2}$. This track is given by the homotopy $I S^{2} \rightarrow \Sigma S^{2}=S^{3} \stackrel{x}{\rightarrow}$ $\vee_{A} S^{2}$, where the first map is the obvious projection. The reader can check that $-H o p f(x)$ is the classical Hopf invariant of $x$. The sign is due to the fact that in order to define the Hopf invariant of $x$ as a track we need to consider the map (12): $I_{+} \wedge S^{1} \wedge S^{1} \cong S^{1} \wedge I_{+} \wedge S^{1}$ and this map induces $-1: S^{3} \rightarrow S^{3}$ up to homotopy.

The next results are crucial for this paper.

Theorem 3.5. Let $f, g: \vee_{A} S^{1} \rightarrow \vee_{B} S^{1}$ be two maps and $n \geq 1$. If a nil-track

$$
N_{f, g}: \Sigma^{n-1} f \Rightarrow \Sigma^{n-1} g
$$

exists then it is unique. Moreover, $N_{f, g}$ exists if and only if

- $\pi_{1} f=\pi_{1} g:\langle A\rangle \rightarrow\langle B\rangle$, if $n=1$;

- or $\left(\pi_{1} f\right)_{n i l}=\left(\pi_{1} g\right)_{n i l}:\langle A\rangle_{n i l} \rightarrow\langle B\rangle_{n i l}$, if $n \geq 2$.

Furthermore, trivial tracks are nil-tracks and the vertical and horizontal composition of nil-tracks are also nil-tracks.

This theorem is a immediate consequence of the following one.

Theorem 3.6. Let $n \geq 2$ and let $f, g: \vee_{A} S^{1} \rightarrow \vee_{B} S^{1}$ be maps such that for any $x \in\langle A\rangle_{\text {nil }}$ we have $\left(\pi_{1} g\right)_{\text {nil }}(x)=\left(\pi_{1} f\right)_{\text {nil }}(x)+\partial \alpha(x)$ for some homomorphism $\alpha: \mathbb{Z}[A] \rightarrow \otimes_{n}^{2} \mathbb{Z}[B]$. Then there exists a unique track $H: \Sigma^{n-1} f \Rightarrow \Sigma^{n-1} g$ with 
Hopf invariant $\operatorname{Hopf}(H)=\alpha$ and conversely. Moreover, the Hopf invariant of tracks satisfies the following formulas. Given a diagram

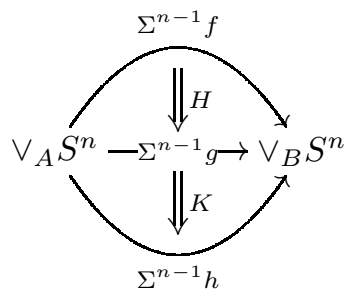

the equation

(1) $\operatorname{Hopf}(K \square H)=\operatorname{Hopf}(K)+\operatorname{Hopf}(H)$ holds.

Furthermore, if we consider the diagram

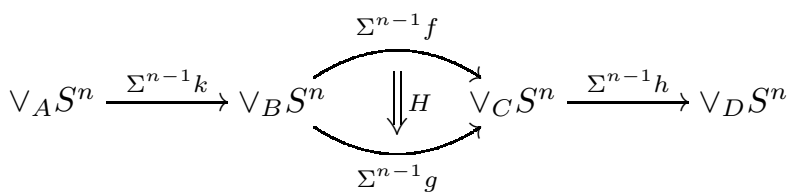

then

(2) $\operatorname{Hopf}\left(H\left(\Sigma^{n-1} k\right)\right)=\operatorname{Hopf}(H)\left(\pi_{1} k\right)_{a b}$,

(3) $\operatorname{Hopf}\left(\left(\Sigma^{n-1} h\right) H\right)=\left(\otimes_{n}^{2}\left(\pi_{1} h\right)_{a b}\right) \operatorname{Hopf}(H)$.

In addition given a track $H: \Sigma^{n-1} f \Rightarrow \Sigma^{n-1} g$ between maps $f, g,: \vee_{A} S^{1} \rightarrow \vee_{B} S^{1}$ one gets the following equations.

(4) $\operatorname{Hopf}(\Sigma H)=0$ if $n=1$,

(5) $\operatorname{Hopf}(\Sigma H)=\bar{\sigma} \operatorname{Hopf}(H)$ if $n=2$,

(6) $\operatorname{Hopf}(\Sigma H)=\operatorname{Hopf}(H)$ if $n \geq 3$.

This theorem is a simple consequence of the theory developed in Bau91 that we now recall.

Let $\mathbf{S}(n) \subset$ Top* be the full track subcategory of one-point unions of $n$-spheres and let $\mathbf{g r}$ be the category of free groups regarded as a track category with only the trivial tracks. Then there is a track functor

$$
\pi_{1}: \mathbf{S}(1) \longrightarrow \mathbf{g r}
$$

given by the fundamental group, $\pi_{1}\left(\vee_{A} S^{1}\right)=\langle A\rangle$. This track functor is a weak equivalence. This follows easily from Bau91 VI.3.13 and the fact that wedges of 1-spheres do not have higher-dimensional homotopy groups.

For $n \geq 2$ we consider the track subcategory $\overline{\mathbf{S}}(n) \subset \mathbf{S}(n)$ of suspended maps. Here objects of $\overline{\mathbf{S}}(n)$ are one-point unions of 1-spheres $\vee_{A} S^{1}$, maps $f, g: \vee_{A}$ $S^{1} \rightarrow \vee_{B} S^{1}$ in $\overline{\mathbf{S}}(n)$ are maps in Top* and tracks $H: f \Rightarrow g$ in $\overline{\mathbf{S}}(n)$ are tracks $H: \Sigma^{n-1} f \Rightarrow \Sigma^{n-1} g$ in Top*. The inclusion

$$
\Sigma^{n-1}: \overline{\mathbf{S}}(n) \subset \mathbf{S}(n)
$$

is given by the $(n-1)$-fold suspension on objects and morphisms and it is the identity on tracks. This is actually a weak equivalence of track categories. See Bau91 VI.4.7.

We now consider the algebraic track category $\operatorname{nil}(n)$ defined as follows. Objects and morphisms are the same as in nil. A track $\alpha: \varphi \Rightarrow \psi$ between homomorphisms 
$\varphi, \psi:\langle A\rangle_{n i l} \rightarrow\langle B\rangle_{n i l}$ is a homomorphism $\alpha: \mathbb{Z}[A] \rightarrow \otimes_{n}^{2} \mathbb{Z}[B]$ such that $\varphi(x)+$ $\partial \alpha(\{x\})=\psi(x)$ for any $x \in\langle A\rangle_{n i l}$. The vertical composition is given by addition of abelian group homomorphisms, and for the horizontal composition one uses the abelianization functor $a b: \mathbf{n i l} \rightarrow \mathbf{a b}$ and the bifunctor

$$
\operatorname{Hom}_{\mathbf{a b}}\left(-, \otimes_{n}^{2}\right): \mathbf{a b}^{o p} \times \mathbf{a b} \longrightarrow \mathbf{A b} .
$$

For any $n \geq 2$ there is a weak equivalence of track categories

$$
\text { Hopf : } \overline{\mathbf{S}}(n) \longrightarrow \operatorname{nil}(n)
$$

defined by $\vee_{A} S^{1} \mapsto\langle A\rangle_{n i l}, f \mapsto\left(\pi_{1} f\right)_{n i l}$ and $H \mapsto H o p f(H)$, where we use the Hopf invariant for tracks. Compare Bau91 VI.4.7. This weak equivalence is compatible on the left hand side with the suspension functor

$$
\Sigma: \overline{\mathbf{S}}(n) \longrightarrow \overline{\mathbf{S}}(n+1)
$$

which is the identity on objects and morphisms and on tracks it is given by the suspension of tracks in Top*, and on the right hand side with the track functors

$$
\operatorname{gr} \longrightarrow \operatorname{nil}(2) \longrightarrow \operatorname{nil}(n), \quad n \geq 3
$$

given by the nilization and the natural projection $\bar{\sigma}: \otimes^{2} \rightarrow \hat{\otimes}^{2}$ respectively. Here we set $\overline{\mathbf{S}}(1)=\mathbf{S}(1)$ for $n=1$. Theorem [3.6] and therefore Theorem 3.5 follows readily from this.

\section{Secondary homotopy groups of a pointed space}

We now introduce secondary homotopy groups which enrich the structure of the classical homotopy groups $\pi_{n} X$ of a pointed space.

Definition 4.1. Let $X$ be a pointed space and $n \geq 1$. The secondary homotopy group $\pi_{n, *} X$ is the map

$$
\partial: \pi_{n, 1} X \longrightarrow \pi_{n, 0} X
$$

defined as follows. Let

$$
\pi_{n, 0} X= \begin{cases}\langle\Omega X\rangle, & n=1 ; \\ \left\langle\Omega^{n} X\right\rangle_{n i l}, & n \geq 2 .\end{cases}
$$

Here the $n$-fold loop space, regarded as a discrete pointed set, generates a free (nilgroup. Moreover, $\pi_{n, 1} X$ is the set of equivalence classes $[f, F]$ represented by a map $f: S^{1} \rightarrow \vee_{\Omega^{n} X} S^{1}$ and a track

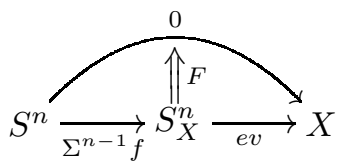

Here the pointed space

$$
S_{X}^{n}=\vee_{\Omega^{n} X} S^{n}=\Sigma^{n} \Omega^{n} X
$$

is the $n$-fold suspension of the $n$-fold loop space $\Omega^{n} X$, where $\Omega^{n} X$ is regarded as a pointed set with the discrete topology. Hence $S_{X}^{n}$ is the coproduct of $n$-spheres indexed by the set of non-trivial maps $S^{n} \rightarrow X$, and $e v: S_{X}^{n} \rightarrow X$ is the obvious evaluation map. Moreover, for the sake of simplicity given a map $f: S^{1} \rightarrow \vee_{\Omega^{n}} S^{1}$ we will denote $f_{e v}=e v\left(\Sigma^{n-1} f\right)$, so that $F$ in the previous diagram is a track $F: f_{e v} \Rightarrow 0$. The equivalence relation $[f, F]=[g, G]$ holds provided the nil-track 
$N_{f, g}: \Sigma^{n-1} f \Rightarrow \Sigma^{n-1} g$ exists, see Theorem 3.5] and the composite track in the following diagram is the trivial track.

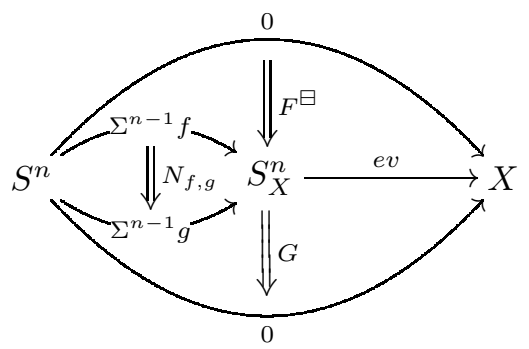

That is $F=G \square\left(e v N_{f, g}\right)$. The map $\partial$ is defined by the formula

$$
\partial[f, F]= \begin{cases}\left(\pi_{1} f\right)(1), & n=1, \\ \left(\pi_{1} f\right)_{n i l}(1), & n \geq 2,\end{cases}
$$

where $1 \in \pi_{1} S^{1}=\mathbb{Z}$.

A map $g: X \rightarrow Y$ in Top* induces a map $\pi_{n, *} g: \pi_{n, *} X \rightarrow \pi_{n, *} Y$ given by the following commutative diagram.

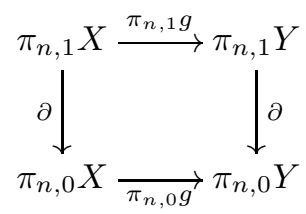

Here the lower homomorphism $\pi_{n, 0} g=\left\langle\Omega^{n} g\right\rangle$ is induced by the map of pointed sets $\Omega^{n} g: \Omega^{n} X \rightarrow \Omega^{n} Y$. Moreover, an element $[f, F] \in \pi_{n, 1} X$ is sent by the upper map $\pi_{n, 1} g$ to $\left[\left(\Sigma \Omega^{n} g\right) f, g F\right]$

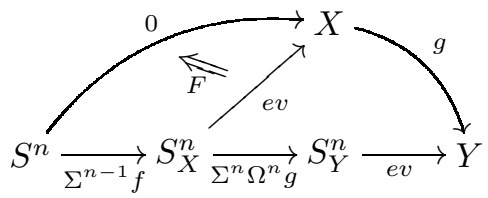

We also define $\pi_{n, *} X$ for $n=0$ as follows.

Definition 4.3. For $n=0$ let $\pi_{0, *} X$ be the fundamental pointed groupoid of the pointed space $X$ for which $\pi_{0,0} X$ is $X$ regarded as a discrete pointed set and $\pi_{0,1} X$ is the set of tracks between points in $X$. For this we recall that a pointed groupoid is a small category $\mathbf{G}$ with a distinguished object $* \in O b \mathbf{G}$ such that all morphisms are isomorphisms. A morphism of pointed groupoids $F: \mathbf{G} \rightarrow \mathbf{H}$ is a functor preserving the distinguished object $F(*)=*$, and the category of pointed groupoids is denoted by grd $^{*}$. The morphism $F$ is a weak equivalence if it induces a bijection between the pointed sets of isomorphism classes of objects $\operatorname{Iso}(F): \operatorname{Iso}(\mathbf{G}) \cong \operatorname{Iso}(\mathbf{H})$ and if $F: \operatorname{Aut}_{\mathbf{G}}(x) \cong \operatorname{Aut}_{\mathbf{H}}(F(x))$ is an isomorphism for any object $x$ in $\mathbf{G}$. The fundamental groupoid is a functor $\pi_{0, *}: \mathbf{T o p}^{*} \rightarrow \mathbf{g r d}^{*}$ in the obvious way.

We now study the algebraic structure of secondary homotopy groups $\pi_{n, *} X$ with $n \geq 1$. 
Proposition 4.4. For $n \geq 1$ there is a group structure on $\pi_{n, 1} X$ such that the map $\partial: \pi_{n, 1} X \rightarrow \pi_{n, 0} X$ is a homomorphism. Moreover, the rows in (4.2) are also group homomorphisms.

Proof. We define the sum of two elements $[f, F],[g, G] \in \pi_{n, 1} X$ by the following diagram

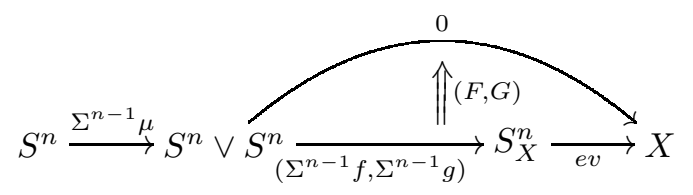

i. e.

$$
[f, F]+[g, G]=\left[(f, g) \mu,(F, G)\left(\Sigma^{n-1} \mu\right)\right] .
$$

One can readily check by using Theorem 3.5 that this operation is associative and $\left[0,0^{\square}\right]$ is a unit element. The inverse of an element $[f, F]$ is represented by

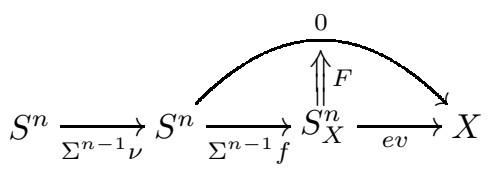

i. e.

$$
-[f, F]=\left[f \nu, F\left(\Sigma^{n-1} \nu\right)\right]
$$

To see this, and in order to introduce the reader to "track arguments", we observe that the following composite tracks are the same

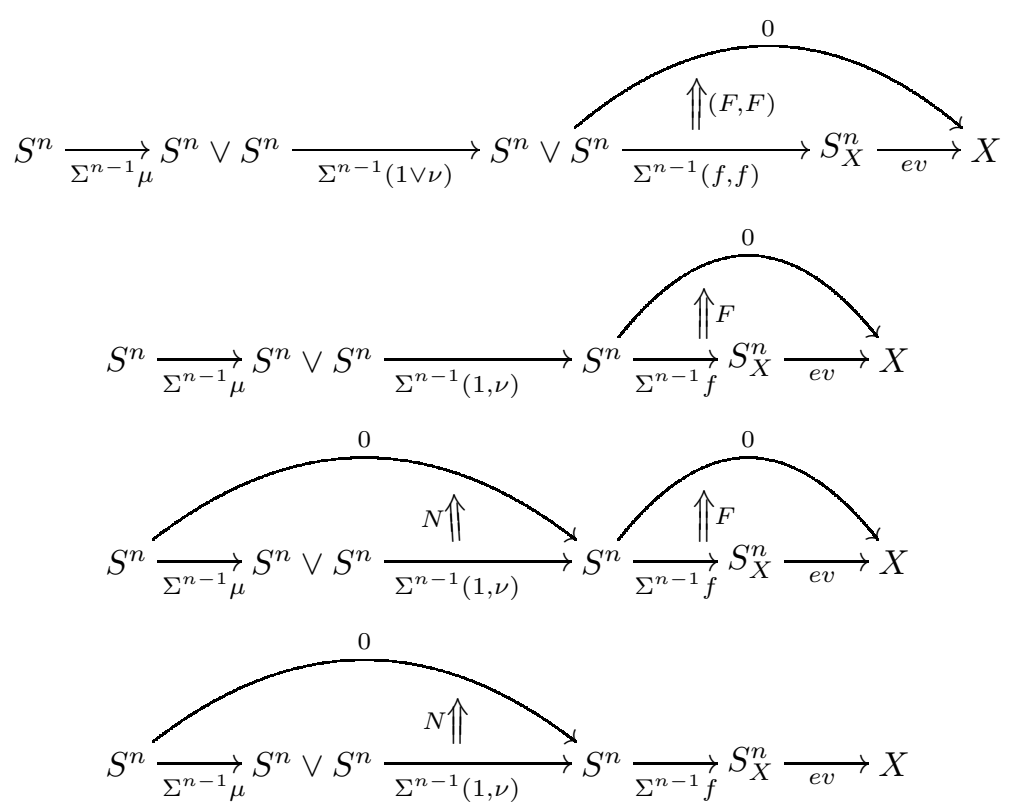

Here the last diagram represents by definition the trivial element in $\pi_{n, 1} X$.

The reader can now easily check that $\partial$ is indeed a homomorphism.

Definition 4.5. A crossed module $\partial: M \rightarrow N$ is a group homomorphism such that $N$ acts on the right of $M$ (the action will be denoted exponentially) and the homomorphism $\partial$ satisfies the following two properties $\left(m, m^{\prime} \in M, n \in N\right)$ : 
(1) $\partial\left(m^{n}\right)=-n+\partial(m)+n$,

(2) $m^{\partial\left(m^{\prime}\right)}=-m^{\prime}+m+m^{\prime}$.

A morphism $\left(f_{0}, f_{1}\right): \partial \rightarrow \partial^{\prime}$ between crossed modules $\partial: M \rightarrow N$ and $\partial^{\prime}: M^{\prime} \rightarrow$ $N^{\prime}$ is a commutative square in the category of groups

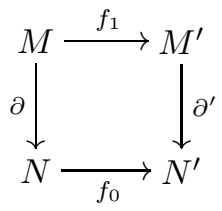

such that for any $m \in M$ and $n \in N$ the formula $f_{1}\left(m^{n}\right)=f_{1}(m)^{f_{0}(n)}$ holds. Such a morphism is a weak equivalence if it induces isomorphisms Ker $\partial \cong \operatorname{Ker} \partial^{\prime}$ and Coker $\partial \cong$ Coker $\partial^{\prime}$. The category of crossed modules will be denoted by cross. A crossed module $\partial: M \rightarrow N$ is 0 -free if $N=\langle E\rangle$ is a free group.

Proposition 4.6. The group $\pi_{1,0} X$ acts on $\pi_{1,1} X$ in such a way that $\partial: \pi_{1,1} X \rightarrow$ $\pi_{1,0} X$ is a crossed module. Moreover, the induced map $\pi_{1, *} g$ in 4.2$)$ is a crossed module morphism.

Proof. Let $\alpha: S^{1} \rightarrow S^{1} \vee S^{1}$ be any map inducing $\pi_{1} \alpha: \mathbb{Z} \rightarrow\langle a, b\rangle: 1 \mapsto-a+b+a$. Any $x \in \pi_{1,0} X$ can be identified with the homotopy class of a map $\tilde{x}: S^{1} \rightarrow S_{X}^{1}$. The automorphism

$$
(-)^{x}: \pi_{1,1} X \longrightarrow \pi_{1,1} X:[f, F] \mapsto[f, F]^{x}
$$

is defined as follows: Let $[f, F]^{x}$ be given by the map

$$
S^{1} \stackrel{\alpha}{\longrightarrow} S^{1} \vee S^{1} \stackrel{(\tilde{x}, f)}{\longrightarrow} S_{X}^{1}
$$

and the track

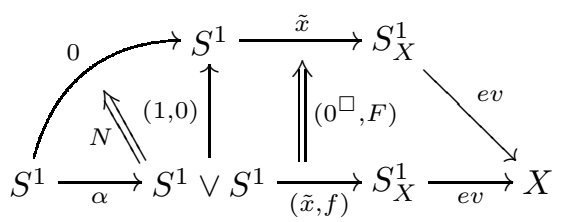

Here $N$ is a nil-track. By using the elementary properties of nil-tracks in Theorem 3.5 the reader can check that this is a well-defined action, independent of the choice of $\alpha$. Equation (1) in Definition 4.5 is immediate. Let us now check that (2) holds. Consider $[f, F],[g, G] \in \pi_{1,1} X$. On one hand $[f, F]^{\partial[g, G]}$ is

(a)

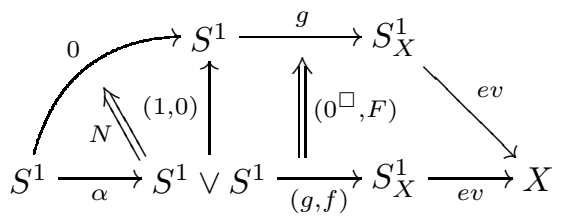

On the other hand $-[g, G]+[f, F]+[g, G]$ is

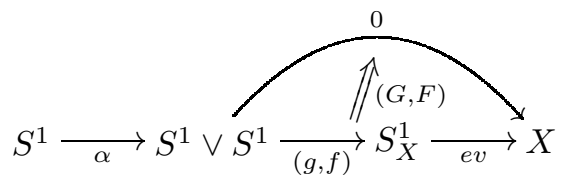


Diagram (b) is obviously the same as

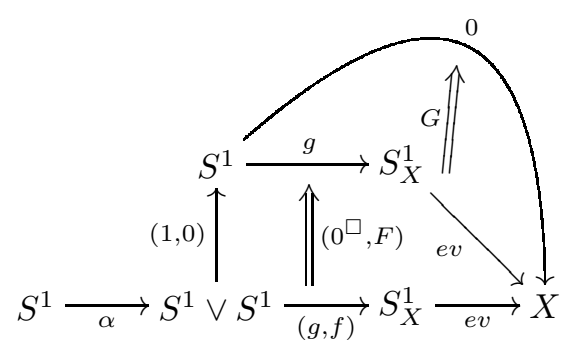

Now we observe that (a) and (c) coincide, hence we are done.

Definition 4.7. A reduced quadratic module $(\omega, \partial)$ is a sequence of group homomorphisms

$$
N_{a b} \otimes N_{a b} \stackrel{\omega}{\longrightarrow} M \stackrel{\partial}{\longrightarrow} N
$$

such that, if $N \rightarrow N_{a b}: x \mapsto\{x\}$ is the projection onto the abelianization, then the following equations hold for any $x, y \in N$ and $a, b \in M$,

(1) $\partial \omega(\{x\} \otimes\{y\})=-x-y+x+y$,

(2) $\omega(\{\partial a\} \otimes\{\partial b\})=-a-b+a+b$,

(3) $\omega(\{\partial a\} \otimes\{x\}+\{x\} \otimes\{\partial a\})=0$.

Moreover, it is a stable quadratic module if the following condition, stronger than (3), is satisfied,

(4) $\omega(\{x\} \otimes\{y\}+\{y\} \otimes\{x\})=0$.

Condition (4) says that $\omega: \otimes^{2} N_{a b} \rightarrow M$ in a stable quadratic module factors through the natural projection $\bar{\sigma}: \otimes^{2} N_{a b} \rightarrow \hat{\otimes}^{2} N_{a b}$. The factorization will also be denoted by $\omega: \hat{\otimes}^{2} N_{a b} \rightarrow M$. We call $(\omega, \partial: M \rightarrow N) 0$-free if $N=\langle E\rangle_{n i l}$ is a free nil-group.

A morphism of reduced or stable quadratic modules $\left(f_{1}, f_{0}\right):(\omega, \partial) \rightarrow\left(\omega^{\prime}, \partial^{\prime}\right)$ is just a commutative diagram of the form

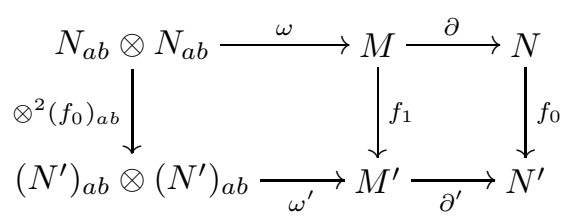

It is a weak equivalence if it induces isomorphisms Ker $\partial \cong \operatorname{Ker} \partial^{\prime}$ and Coker $\partial \cong$ Coker $\partial^{\prime}$. We will write rquad and squad for the categories of reduced and symmetric quadratic modules, respectively. See [Bau91] IV.C.1.

We now define a map $\omega$ as in Definition 4.7 for secondary homotopy groups $\pi_{n, *} X$ with $n \geq 2$. Consider the diagram

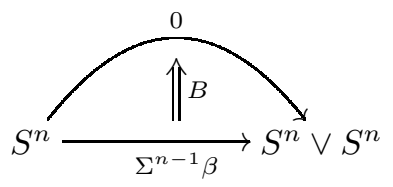

where $\beta: S^{1} \rightarrow S^{1} \vee S^{1}$ is given such that $\left(\pi_{1} \beta\right)_{\text {nil }}(1)=-a-b+a+b \in\langle a, b\rangle_{\text {nil }}$ is the commutator. The track $B$ is determined by $\operatorname{Hopf}(B)=-a \otimes b \in \otimes^{2} \mathbb{Z}[a, b]$. 
Given $x \otimes y \in \otimes_{n}^{2}\left(\pi_{n, 0} X\right)_{a b}$ let $\tilde{x}, \tilde{y}: S^{1} \rightarrow \vee_{\Omega^{n} X} S^{1}$ be maps with $\left(\pi_{1} \tilde{x}\right)_{a b}(1)=x$ and $\left(\pi_{1} \tilde{y}\right)_{a b}(1)=y$. Then the diagram

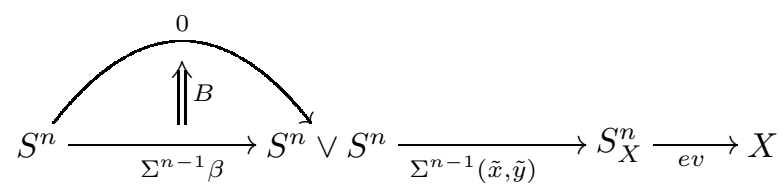

represents an element

$$
\omega(x \otimes y)=\left[(\tilde{x}, \tilde{y}) \beta, e v\left(\Sigma^{n-1}(\tilde{x}, \tilde{y})\right) B\right] \in \pi_{n, 1} X .
$$

Proposition 4.9. For $n \geq 2$ the homomorphism of groups

$$
\omega:\left(\pi_{n, 0} X\right)_{a b} \otimes\left(\pi_{n, 0} X\right)_{a b} \longrightarrow \pi_{n, 1} X
$$

given by 4.8$)$ is well defined. Moreover, $(\omega, \partial)$ is a reduced quadratic module for $n=2$ and a stable quadratic module for $n \geq 3$. Furthermore, (4.2) is a reduced quadratic module homomorphism for all $n \geq 2$.

Proof. Given $x \in \otimes_{n}^{2}\left(\pi_{n, 0} X\right)_{a b}$ we alternatively define

$$
\omega(x)=\left[\omega(x)_{1}, e v \omega(x)_{2}\right] \in \pi_{n, 1} X
$$

by choosing $\omega(x)_{1}$ and $\omega(x)_{2}:\left(\omega(x)_{1}\right)_{e v} \Rightarrow 0$ as in the diagram

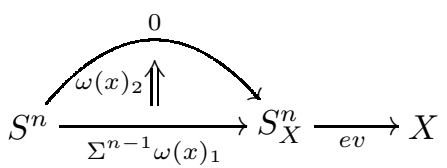

Here $\omega(x)_{1}: S^{1} \rightarrow \vee_{\Omega^{n} X} S^{1}$ is a map with $\left(\pi_{1} \omega(x)_{1}\right)_{n i l}(1)=\partial(x)$ and

$$
\omega(x)_{2}: \Sigma^{n-1} \omega(x)_{1} \Rightarrow 0
$$

is the unique track with $\operatorname{Hopf}\left(\omega(x)_{2}\right)=-x$. Such a track exists and is unique by Theorem 3.6. The elementary properties of nil-tracks and more generally of the Hopf invariant for tracks, see Theorem [3.6. show that the element $\omega(x)$ is indeed well-defined and this definition of $\omega$ coincides with the definition given by (4.8). Axiom (1) in Definition 4.7 is automatically satisfied. The bilinearity of $\omega$ follows from Theorem 3.6 (1) and the following claim:

$\left(^{*}\right)$ Given $[f, F] \in \pi_{1, n} X$ and $x \in \otimes_{n}^{2}\left(\pi_{n, 0} X\right)_{a b}$ then the sum $[f, F]+\omega(x)=$ $[g, G] \in \pi_{n, 1} X$ is represented by a map $g$ with $\left(\pi_{1} g\right)_{n i l}(1)=\partial[f, F]+\partial(x)$ and $G=F \square(e v \bar{G})$ where $\bar{G}: \Sigma^{n-1} g \Rightarrow \Sigma^{n-1} f$ is the unique track with $\operatorname{Hopf}(\bar{G})=-x$.

Indeed $[f, F]+\omega(x)=\left[\left(f, \omega(x)_{1}\right) \mu,\left(F, e v \omega(x)_{2}\right)\left(\Sigma^{n-1} \mu\right)\right]$ and we can suppose $g=$ $\left(f, \omega(x)_{1}\right) \mu$. The following composite track is clearly trivial

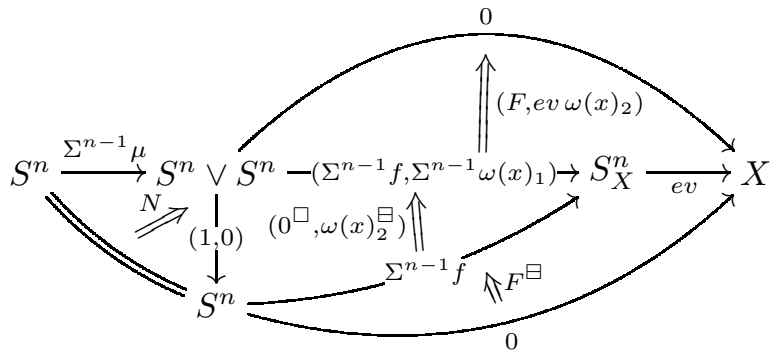


Here $N$ is a nil-track. Moreover, it is not difficult to see by using Theorem 3.6 that

$$
\operatorname{Hopf}\left(\left(\left(\Sigma^{n-1} f\right) N^{\boxminus}\right) \square\left(\left(0^{\square}, \omega(x)_{2}\right)\left(\Sigma^{n-1} \mu\right)\right)\right)=-x .
$$

therefore $\bar{G}=\left(\left(\Sigma^{n-1} f\right) N^{\boxminus}\right) \square\left(\left(0^{\square}, \omega(x)_{2}\right)\left(\Sigma^{n-1} \mu\right)\right)$ and the claim follows.

It is easy to see that the commutator $-[f, F]-[g, G]+[f, F]+[g, G] \in \pi_{n, 1} X$ is given by the following diagram

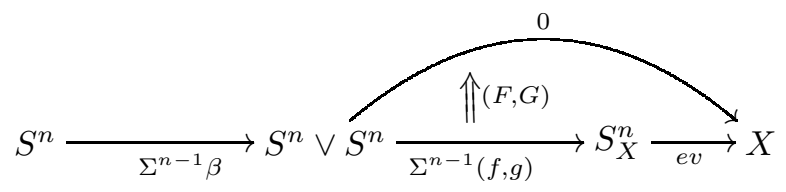

This diagram coincides with

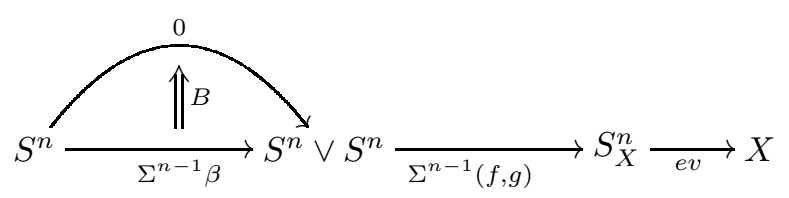

Therefore (2) in Definition 4.7] is satisfied.

If $n \geq 3$ equation (3) in Definition 4.7 is an immediate consequence of the fact that $\bar{\sigma}(\{x\} \otimes\{y\}+\{y\} \otimes\{x\})=0$. Let us now check (3) in Definition 4.7 in case $n=2$. Suppose that we have $[f, F] \in \pi_{2,1} X$ and $x \in \pi_{2,0} X$. We choose $\tilde{x}: S^{1} \rightarrow \vee_{\Omega^{2} X} S^{1}$ such that $\left(\pi_{1} \tilde{x}\right)_{\text {nil }}(1)=x$. Then by claim $\left(^{*}\right)$ and (4.8) we have that $\omega(\{\partial[f, F]\} \otimes\{x\}+\{x\} \otimes\{\partial[f, F]\})$ is represented by the diagram

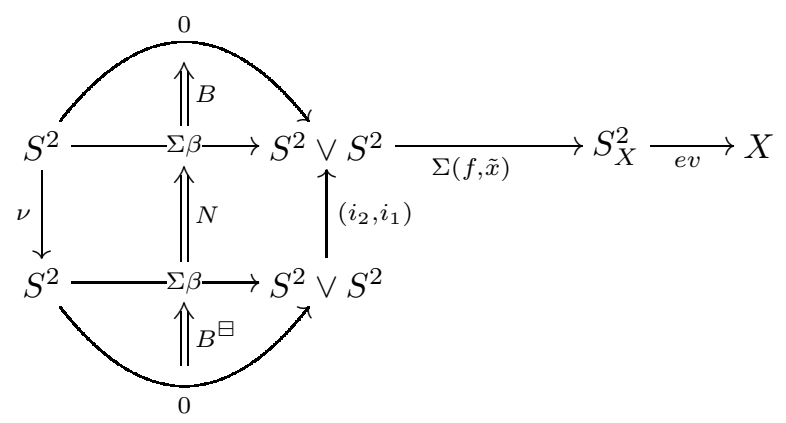

Here $N$ is a nil-track. This diagram coincides with

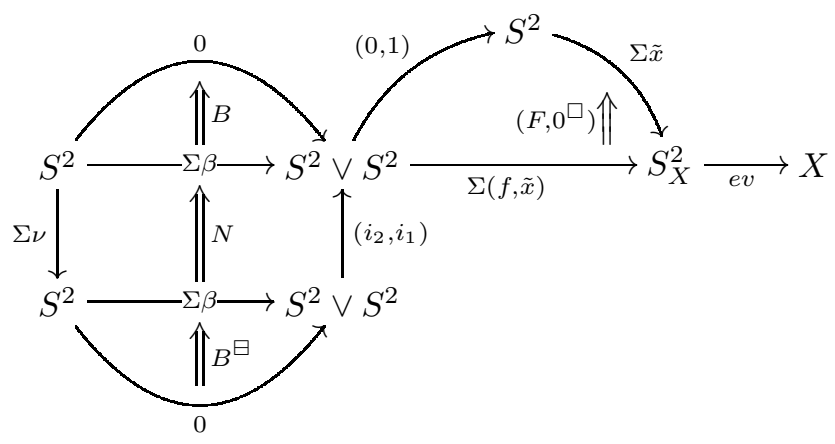


and this one is the same as

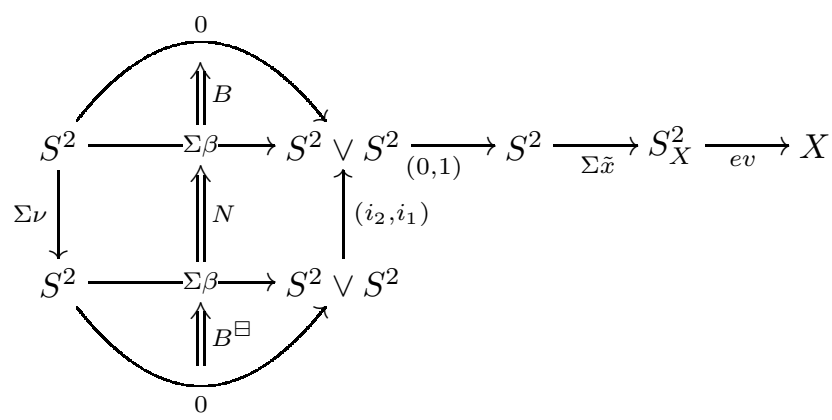

By using Theorem 3.6 one can readily check that $(0,1)\left(B \square N \square\left(\left(i_{2}, i_{1}\right) B^{\boxminus}(\Sigma \nu)\right)\right)$ is a nil-track, and therefore this diagram represents the trivial element in $\pi_{2,1} X$.

For $n \geq 0$ we define the category $\operatorname{cross}(n)$ as follows.

$$
\operatorname{cross}(n)= \begin{cases}\operatorname{grd}^{*}, & \text { pointed groupoids if } n=0 ; \\ \text { cross, } & \text { crossed modules if } n=1 ; \\ \text { rquad, } & \text { reduced quadratic modules if } n=2 ; \\ \text { squad, } & \text { stable quadratic modules if } n \geq 3 .\end{cases}
$$

Theorem 4.11. Secondary homotopy groups are well-defined functors

$$
\pi_{n, *}: \text { Top }^{*} \longrightarrow \operatorname{cross}(n), \quad n \geq 0 .
$$

This result generalizes the well-known fact on classical homotopy groups which are functors

$$
\pi_{n}: \mathbf{T o p}^{*} \longrightarrow \operatorname{group}(n), \quad n \geq 0
$$

where

$$
\operatorname{group}(n)= \begin{cases}\text { Set }^{*}, & \text { pointed sets if } n=0 ; \\ \mathbf{G r}, & \text { groups if } n=1 ; \\ \mathbf{A b}, & \text { abelian groups if } n \geq 2 .\end{cases}
$$

Moreover, we have functors $(n \geq 0)$

$$
\begin{gathered}
h_{0}: \operatorname{cross}(n) \longrightarrow \operatorname{group}(n), \\
h_{1}: \operatorname{cross}(n) \longrightarrow \operatorname{group}(n+1) .
\end{gathered}
$$

The functor $h_{0}$ is defined as the cokernel of the group homomorphism $\partial: M \rightarrow N$ for a crossed module $\partial$ or a reduced or stable quadratic module $(\omega, \partial)$, and for $\mathbf{G}$ a pointed groupoid $h_{0} \mathbf{G}=\operatorname{Iso}(\mathbf{G})$ is the pointed set of isomorphism classes of objects. Similarly $h_{1}$ is the kernel of $\partial: M \rightarrow N$ for crossed modules and reduced and stable quadratic modules, and $h_{1} \mathbf{G}=\operatorname{Aut}_{\mathbf{G}}(*)$ is the automorphism group of the distinguished object. In particular a morphism $f$ in $\operatorname{cross}(n)$ is a weak equivalence for $n \geq 1$ if and only if $h_{0} f$ and $h_{1} f$ are isomorphisms.

In Proposition 5.1 below we show that there are natural isomorphisms $(n \geq 0)$

$$
h_{0} \pi_{n, *} X \cong \pi_{n} X \text { and } h_{1} \pi_{n, *} X \cong \pi_{n+1} X .
$$


Our definition of $\pi_{n, *} X$ above is a "singular" and hence functorial version of secondary homotopy groups. For many purposes it suffices to consider smaller models of $\pi_{n, *} X$ by choosing a subset of $\Omega^{n} X$ which generates $\pi_{n} X$ as an abelian group. Let us make precise this observation.

Proposition 4.15. Let $X$ be a pointed space. If $E_{0} \rightarrow X$ is a pointed map between pointed sets then there is a unique pointed groupoid $\pi_{0, *}\left(X, E_{0}\right)$ with object set $E_{0}$ endowed with a full and faithful functor

$$
\pi_{0, *}\left(X, E_{0}\right) \longrightarrow \pi_{0, *} X
$$

given by $E_{0} \rightarrow X$ on object sets. This morphism of pointed groupoids is a weak equivalence provided any component of $X$ has points in the image of $E_{0}$. Moreover, a map of pointed sets $E_{1} \rightarrow \Omega X$ induces a crossed module morphism by the pull-back

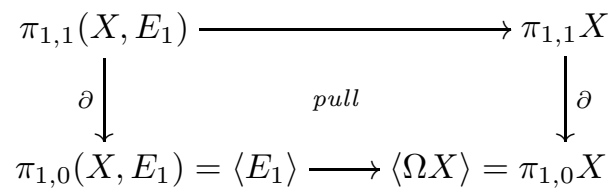

which is a weak equivalence

$$
\pi_{1, *}\left(X, E_{1}\right) \stackrel{\sim}{\longrightarrow} \pi_{1, *} X
$$

provided the loops in the image of $E_{1}$ generate the group $\pi_{1} X$. Furthermore, for $n \geq 2$ the a map of pointed sets $E_{n} \rightarrow \Omega^{n} X$ induces a reduced (stable if $n \geq 3$ ) quadratic module morphism by the pull-back

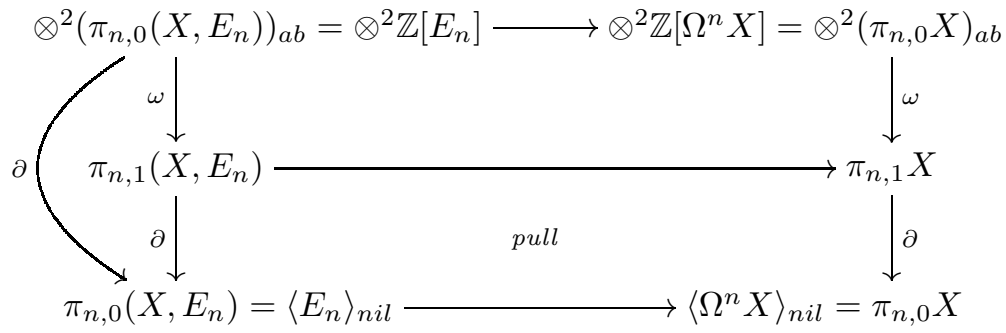

which is a weak equivalence

$$
\pi_{n, *}\left(X, E_{n}\right) \stackrel{\sim}{\longrightarrow} \pi_{n, *} X, n \geq 2
$$

provided the $n$-loops in the image of $E_{n}$ generate the abelian group $\pi_{n} X$.

This proposition can be used to reduce the number of generators of a secondary homotopy group, as one can check in the following example.

Remark 4.16. So far we have not computed any secondary homotopy group. Now, with the help of Proposition 4.15 we give a small model for the secondary homotopy group $\pi_{n, *}\left(\vee_{E} S^{n}\right)$ of a wedge of spheres indexed by the pointed set $E$. For this we notice that there is a pointed inclusion $E \subset \Omega^{n}\left(\vee_{E} S^{n}\right)$ sending $e \in E-\{*\}$ to the inclusion of the corresponding factor of the wedge $S^{n} \subset \vee_{E} S^{n}$. Then we have a weak equivalence

$$
\pi_{n, *}\left(\vee_{E} S^{n}, E\right) \stackrel{\sim}{\longrightarrow} \pi_{n, *}\left(\vee_{E} S^{n}\right), \quad n \geq 1 .
$$

For $n=1$ one easily checks that $\pi_{1, *}\left(\vee_{E} S^{1}, E\right)$ is

$$
\pi_{1,1}\left(\vee_{E} S^{1}, E\right)=0 \stackrel{\partial}{\longrightarrow} \pi_{1,0}\left(\vee_{E} S^{1}, E\right)=\langle E\rangle
$$


For $n=2$ the reduced quadratic module $\pi_{2, *}\left(\vee_{E} S^{2}, E\right)$ is given by the following diagram, see (2.2)

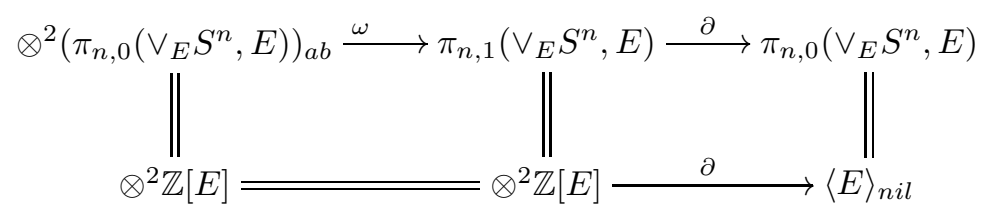

This follows from the fact that the next diagram is a pull-back

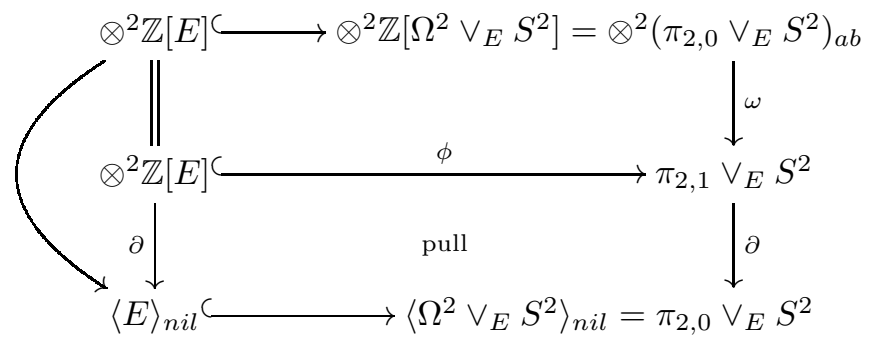

Here the homomorphism $\phi$ is defined as follows. Given $x \in \otimes^{2} \mathbb{Z}[E]$ the element $\phi(x)=\left[\phi_{1}(x), \phi_{2}(x)\right]$ is given by a map

$$
\phi_{1}(x): S^{1} \stackrel{\bar{\phi}(x)}{\longrightarrow} \vee_{E} S^{1} \subset \vee_{\Omega^{2} \vee_{E} S^{2}} S^{1}
$$

with $\left(\pi_{1} \bar{\phi}(x)\right)_{n i l}(1)=\partial(x)$ and the unique track

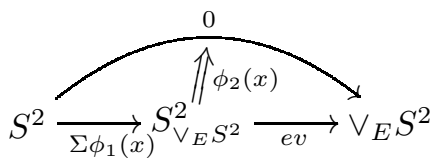

with Hopf invariant $\operatorname{Hopf}\left(\phi_{2}(x)\right)=-x$. Here we use the fact that the composite $e v\left(\Sigma \phi_{1}(x)\right)=\Sigma \bar{\phi}(x)$ is a suspension. For $n \geq 3$ the stable quadratic module $\pi_{n, *}\left(\vee_{E} S^{n}, E\right)$ is given by the following diagram, see (2.2).

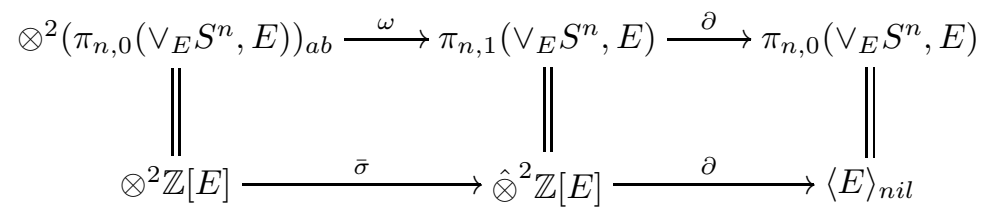

This can be easily checked as in the case $n=2$ by using the Hopf invariant for tracks.

\section{Homotopy Groups OF FIBERS}

We first obtain by secondary homotopy groups the classical homotopy groups $\pi_{n} X$ as in the next result.

Proposition 5.1. For all $n \geq 1$ there is a natural exact sequence of groups

$$
\pi_{n+1} X \stackrel{\iota}{\hookrightarrow} \pi_{n, 1} X \stackrel{\partial}{\longrightarrow} \pi_{n, 0} X \stackrel{q}{\rightarrow} \pi_{n} X,
$$

where $q$ sends a basis element of $\pi_{n, 0} X$, which is a map $f: S^{n} \rightarrow X$, to its homotopy class in $\pi_{n} X$; and $\iota$ carries the homotopy class of $f: S^{n+1} \rightarrow X$ to the element $[0, p f] \in \pi_{n, 1} X$, where $p: I S^{n} \rightarrow \Sigma S^{n}=S^{n+1}$ is the obvious projection. 
Proof. Obviously $q$ is surjective. Any element $x \in \pi_{n, 0} X$ is represented by a map $\tilde{x}: S^{1} \rightarrow \vee_{\Omega^{n} X} S^{1}$, i. e. $\left(\pi_{1} \tilde{x}\right)_{n i l}(1)=x$. It is immediate to notice that $q(x)$ is the homotopy class of $\tilde{x}_{e v}: S^{n} \rightarrow X$. If $q(x)=0$ then there exists a track $H: \tilde{x}_{e v} \Rightarrow 0$, and the pair $[\tilde{x}, H] \in \pi_{n, 1} X$ satisfies $\partial[\tilde{x}, H]-q(x)$. It is immediate to notice that $q \partial=0$ and $\partial \iota=0$. The injectivity of $\iota$ is also easy to check, actually $\pi_{n+1} X$ is isomorphic to the subgroup of $\pi_{n, 1} X$ given by the elements which can be represented with a 0 in the first coordinate. Finally suppose that for some $[f, F] \in \pi_{n, 1} X$ we have $\partial[f, F]=0$, then the nil-track $N: 0 \Rightarrow \Sigma^{n-1} f$ is defined and $[f, F]=[0, F \square N]$, hence we are done.

We now introduce the (algebraic) fiber of a map in $\operatorname{cross}(n)$ for $n \geq 1$.

Definition 5.2. Let $f: \partial \rightarrow \partial^{\prime}$ be a crossed module morphism

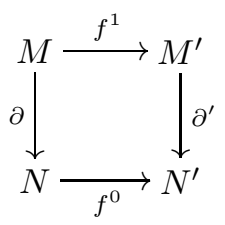

We define the fiber $\operatorname{Fib}(f)$ as the crossed module $\operatorname{Fib}(f): \operatorname{Fib}_{1}(f) \rightarrow \operatorname{Fib}_{0}(f)$ where $\mathrm{Fib}_{0}(f)$ is the following pull-back

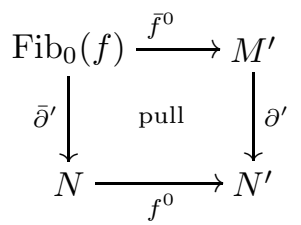

$\operatorname{Fib}_{1}(f)=M$ and the homomorphism $\operatorname{Fib}(f): \operatorname{Fib}_{1}(f) \rightarrow \operatorname{Fib}_{0}(f)$ is induced by $\left(\partial, f^{1}\right): M \rightarrow N \times M^{\prime}$. The action of $\operatorname{Fib}_{0}(f)$ on $\operatorname{Fib}_{1}(f)$ is the pull-back along $\bar{\partial}^{\prime}$ of the action of $N$ on $M$. The axioms of a crossed module are easily verified. There is also a natural crossed module morphism $\jmath: \operatorname{Fib}(f) \rightarrow \partial$ given by the square

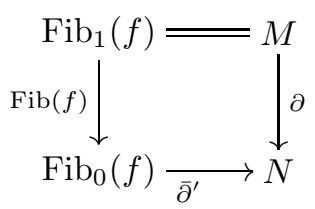

Let $f:(\omega, \partial) \rightarrow\left(\omega^{\prime}, \partial^{\prime}\right)$ be now a reduced/stable quadratic module morphism

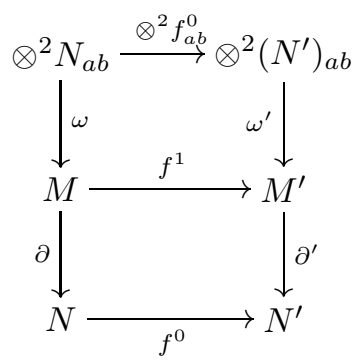


The fiber $\operatorname{Fib}(f)$ is a reduced/stable quadratic module

$$
\otimes^{2}\left(\operatorname{Fib}_{0}(f)\right)_{a b} \longrightarrow \operatorname{Fib}_{1}(f) \stackrel{\operatorname{Fib}(f)}{\longrightarrow} \operatorname{Fib}_{0}(f)
$$

where $\operatorname{Fib}(f): \operatorname{Fib}_{1}(f) \longrightarrow \operatorname{Fib}_{0}(f)$ is defined as in the crossed module case and the first homomorphism is the composite

$$
\otimes^{2}\left(\operatorname{Fib}_{0}(f)\right)_{a b} \stackrel{\otimes^{2} \bar{\partial}_{a b}^{\prime}}{\longrightarrow} \otimes^{2} N_{a b} \stackrel{\omega}{\longrightarrow} M .
$$

The natural reduced/stable quadratic module morphism $\jmath: \operatorname{Fib}(f) \rightarrow(\omega, \partial)$ is also defined as above.

Lemma 5.3. Let $f: \partial \rightarrow \partial^{\prime}$ be a morphism of crossed modules, then there is an exact sequence

$$
h_{1} \operatorname{Fib}(f) \stackrel{h_{1} \jmath}{\longrightarrow} h_{1} \partial \stackrel{h_{1} f}{\longrightarrow} h_{1} \partial^{\prime} \stackrel{\delta}{\longrightarrow} h_{0} \operatorname{Fib}(f) \stackrel{h_{0} \jmath}{\longrightarrow} h_{0} \partial \stackrel{h_{0} f}{\longrightarrow} h_{0} \partial^{\prime} .
$$

This exact sequence is natural in $f$. Moreover, it is also available for reduced or stable quadratic module morphisms $f:(\omega, \partial) \rightarrow\left(\omega^{\prime}, \partial^{\prime}\right)$.

Proof. The homomorphism $\delta$ is determined by the inclusion $M^{\prime} \hookrightarrow N \times M^{\prime}: m^{\prime} \mapsto$ $\left(0, m^{\prime}\right)$. The proof of the exactness is a simple exercise.

Theorem 5.4. Let $f: X \rightarrow Y$ be a map between pointed spaces and let $F_{f}$ be the homotopy fiber of $f$. Then for all $n \geq 1$ there is a natural morphism in $\operatorname{cross}(n)$

$$
\xi: \pi_{n, *} F_{f} \longrightarrow \operatorname{Fib}\left(\pi_{n, *} f\right)
$$

which induces an isomorphism

$$
\pi_{n} F_{f} \cong h_{0} \operatorname{Fib}\left(\pi_{n, *} f\right)
$$

and an exact sequence

$$
\pi_{n+2} Y \longrightarrow \pi_{n+1} F_{f} \rightarrow h_{1} \operatorname{Fib}\left(\pi_{n, *} f\right),
$$

where the first arrow is the boundary homomorphism in the long exact sequence in homotopy. By using the isomorphism (1) above and Proposition [5.1] we can naturally identify the exact sequence in Lemma 5.3 extended on the left by the exact sequence (2) with the following piece of the long exact sequence of homotopy groups

$$
\pi_{n+2} Y \rightarrow \pi_{n+1} F_{f} \rightarrow \pi_{n+1} X \rightarrow \pi_{n+1} Y \rightarrow \pi_{n} F_{f} \rightarrow \pi_{n} X \rightarrow \pi_{n} Y .
$$

Proof. Recall that $F_{f}$ is a pull-back

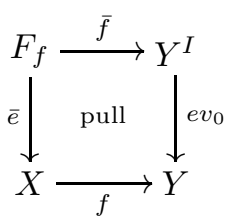

where $Y^{I}$ is the space of based maps $([0,1], 1) \rightarrow(Y, *)$ and $e v_{0}$ is the evaluation at $0 \in[0,1]$.

The morphism $\xi$ consists of two morphisms, the upper one is

$$
\xi^{1}=\pi_{n, 1} \bar{e}: \pi_{n, 1} F_{f} \rightarrow \pi_{n, 1} X=\operatorname{Fib}_{1}\left(\pi_{n, *} f\right) .
$$


We now construct the map $\xi_{0}: \pi_{n, 0} F_{f} \rightarrow \mathrm{Fib}_{0}\left(\pi_{n, *} f\right)$. For this we consider on the one hand the morphism $\pi_{n, 0} \bar{e}: \pi_{n, 0} F_{f} \rightarrow \pi_{n, 0} X$ induced by $f$. On the other hand we define a homomorphism

$$
\bar{\xi}: \pi_{n, 0} F_{f} \rightarrow \pi_{n, 1} Y
$$

as follows: an element $z \in \pi_{n, 0} F_{f}$ is represented by a map $\tilde{z}: S^{1} \rightarrow \vee_{\Omega^{n}} F_{f} S^{1}$ with $\left(\pi_{1} \tilde{z}\right)(1)=z$ if $n=1$ or $\left(\pi_{1} \tilde{z}\right)_{n i l}(1)=z$ if $n \geq 2$. The map

$$
S^{n} \stackrel{\Sigma^{n-1} \tilde{z}}{\longrightarrow} S_{F_{f}}^{n} \stackrel{\Sigma^{n} \Omega^{n}}{\longrightarrow} S_{Y^{I}}^{n} \stackrel{e v}{\longrightarrow} Y^{I}
$$

has an adjoint

$$
\operatorname{ad}\left(\operatorname{ev}\left(\Sigma^{n} \Omega^{n} \bar{f}\right)\left(\Sigma^{n-1} \tilde{z}\right)\right): I S^{n} \longrightarrow Y
$$

this adjoint represents a track $\operatorname{ad}\left(\operatorname{ev}\left(\Sigma^{n} \Omega^{n} \bar{f}\right)\left(\Sigma^{n-1} \tilde{z}\right)\right):\left(\left(\Sigma \Omega^{n}(f \bar{e})\right) \tilde{z}\right)_{e v} \Rightarrow 0$, and

$$
\bar{\xi}(z)=\left[\left(\Sigma \Omega^{n}(f \bar{e})\right) \tilde{z}, \operatorname{ad}\left(\operatorname{ev}\left(\Sigma^{n} \Omega^{n} \bar{f}\right)\left(\Sigma^{n-1} \tilde{z}\right)\right)\right] \in \pi_{n, 1} Y .
$$

It is immediate to check that $\pi_{n, 0} \bar{e}$ and $\bar{\xi}$ define a homomorphism to the pull-back

$$
\xi^{0}=\left(\pi_{n, 0} \bar{e}, \bar{\xi}\right): \pi_{n, 0} F_{f} \longrightarrow \operatorname{Fib}_{0}\left(\pi_{n, *} f\right) .
$$

Now it is easy to check that $\xi$ is indeed a morphism in $\operatorname{cross}(n)$.

By Proposition 5.1 and Lemma 5.3 we obtain from $\xi$ a diagram with exact rows

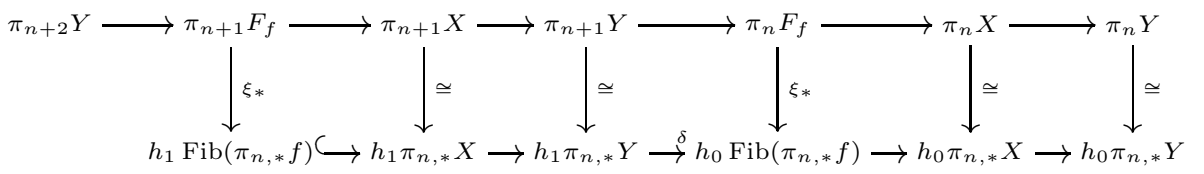

It is easy to see that this diagram commutes, and hence the theorem follows from the five lemma.

Corollary 5.5. Let $f: X \rightarrow Y$ be a map between pointed spaces and let $F_{f}$ be the homotopy fiber of $f$. If $\pi_{n+2} f: \pi_{n+2} X \rightarrow \pi_{n+2} Y$ is surjective then there is a weak equivalence in $\operatorname{cross}(n), n \geq 1$,

$$
\xi: \pi_{n, *} F_{f} \stackrel{\sim}{\longrightarrow} \operatorname{Fib}\left(\pi_{n, *} f\right) .
$$

\section{Suspension And LOOP FUnCtors}

Homotopy groups $\pi_{n} X$ are objects in the category $\operatorname{group}(n), n \geq 0$, see (4.12). There are forgetful functors

$$
\phi_{n}: \operatorname{group}(n) \longrightarrow \operatorname{group}(n-1)
$$

given by $\phi_{n}=1_{\mathbf{A b}}$ for $n \geq 3$ and by the obvious forgetful functors $\phi_{2}: \mathbf{A b} \longrightarrow \mathbf{G r}$, $\phi_{1}: \mathbf{G r} \longrightarrow$ Set $^{*}$.

It is a classical result that for any pointed space $X$ there are natural isomorphisms $n \geq 0$

$$
\Omega: \pi_{n} \Omega X \cong \phi_{n+1} \pi_{n+1} X \text { in } \operatorname{group}(n)
$$

The analogue of this isomorphism for secondary homotopy groups is as follows.

There are forgetful functors

$$
\phi_{n}: \operatorname{cross}(n) \longrightarrow \operatorname{cross}(n-1),
$$


see (4.10), given by $\phi_{n}=1_{\text {squad }}$ for $n \geq 4$ and by the functors

$$
\begin{gathered}
\phi_{3}: \text { squad } \longrightarrow \text { rquad }, \\
\phi_{2}: \text { rquad } \longrightarrow \text { cross }, \\
\phi_{1}: \text { cross } \longrightarrow \text { grd }^{*} .
\end{gathered}
$$

The functor $\phi_{3}$ in (6.3) is obvious, since stable quadratic modules are special reduced quadratic modules. Given a reduced quadratic module $(\omega, \partial)$ we have $\phi_{2}(\omega, \partial)=\partial: M \rightarrow N$ in cross, with the action of $N$ on $M$ defined by

$$
m^{n}=m+\omega(\{\partial m\} \otimes\{n\}) .
$$

Finally if $\partial: M \rightarrow N$ is a crossed module then the pointed groupoid $\phi_{1} \partial$ in grd $^{*}$ has $N$ as a set of objects. Moreover the set of all morphisms in $\phi_{1} \partial$ is the semidirect product $N \ltimes M$, which is the group structure on the set $N \times M$ defined by the formula

$$
(n, m)+\left(n^{\prime}, m^{\prime}\right)=\left(n+n^{\prime}, m^{n^{\prime}}+m^{\prime}\right),
$$

and the structure maps of the groupoid (identities, source and target)

$$
N \stackrel{i}{\rightarrow} N \ltimes M \underset{t}{\stackrel{s}{\rightrightarrows}} N
$$

are $i(n)=(n, 0), s(n, m)=n$ and $t(n, m)=n+\partial m$. The composition law $\circ$ is determined by the formula

$$
\left(n+\partial m, m^{\prime}\right) \circ(n, m)=\left(n, m+m^{\prime}\right) .
$$

The forgetful functors $\phi_{n}$ in (6.2) clearly commute with $h_{0}$ and $h_{1}$ in (4.13), that is,

$$
h_{i} \phi_{n}=\phi_{n} h_{i}, \quad n \geq 1, i=0,1 .
$$

Theorem 6.5. There is a natural weak equivalence in $\operatorname{cross}(n)$

$$
\Omega: \pi_{n, *} \Omega X \longrightarrow \phi_{n+1} \pi_{n+1, *} X, n \geq 0,
$$

which induces the isomorphism $\Omega: \pi_{n} \Omega X \cong \phi_{n+1} \pi_{n+1} X$ in $h_{0}$ and $-\Omega: \pi_{n+1} \Omega X \cong$ $\phi_{n+2} \pi_{n+2} X$ in $h_{1}$. This weak equivalence is an isomorphism for $n \geq 2$.

Proof. Let us first consider the case $n \geq 3$.

We have $\pi_{n, 0} \Omega X=\left\langle\Omega^{n+1} X\right\rangle_{n i l}=\pi_{n+1,0} X$. We define a group homomorphism $\pi_{n, 1} \Omega X \rightarrow \pi_{n+1,1} X$ sending $[f, F]$ with $f: S^{1} \rightarrow \vee_{\Omega^{n+1} X} S^{1}$ and

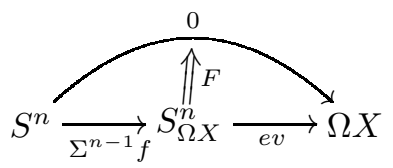

to $[f, a d(F)]$ where $a d(F)$ is the adjoint track

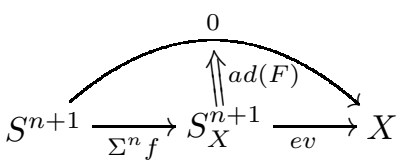

Here we use that $\Sigma S_{\Omega X}^{n}=S_{X}^{n+1}$ and $\operatorname{ad}\left(e v\left(\Sigma^{n-1} f\right)\right)=e v\left(\Sigma^{n} f\right)$. 
The reader can check that the diagram

(a)

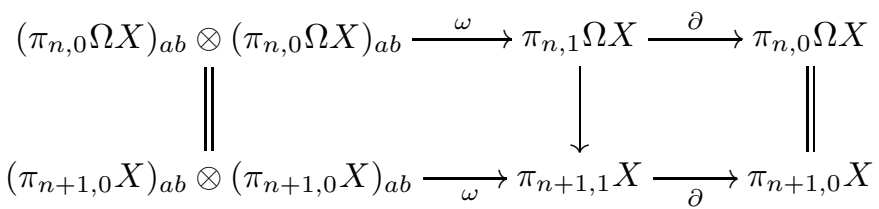

commutes, so it is a morphism of stable quadratic modules. Moreover, the following diagram commutes

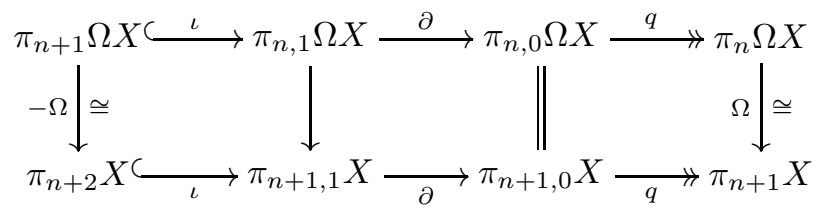

Here the exact rows are given by Proposition 5.1 and the arrows with $\cong$ are (up to sign) the usual isomorphisms of homotopy groups, therefore the central vertical arrow in (a) is an isomorphism by the five lemma.

For $n=2$ we have $\pi_{2,0} \Omega X=\left\langle\Omega^{3} X\right\rangle_{n i l}=\pi_{3,0} X$ and there is a homomorphism $\pi_{2,1} \Omega X \rightarrow \pi_{3,1} X$ defined as above. This homomorphism makes commutative diagrams (a) and (b), therefore it defines an isomorphism of reduced quadratic modules.

For $n=1$ there is an obvious epimorphism $\pi_{1,0} \Omega X=\left\langle\Omega^{2} X\right\rangle \rightarrow\left\langle\Omega^{2} X\right\rangle_{\text {nil }}=$ $\pi_{2,0} X$. One can also define a homomorphism $\pi_{1,1} \Omega X \rightarrow \pi_{2,1} X$ as above. It is easy to check that the following square defines the desired crossed module morphism

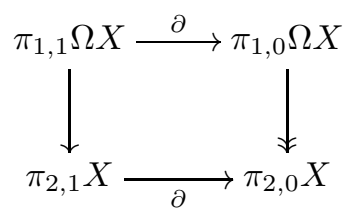

Moreover, the following diagram commutes

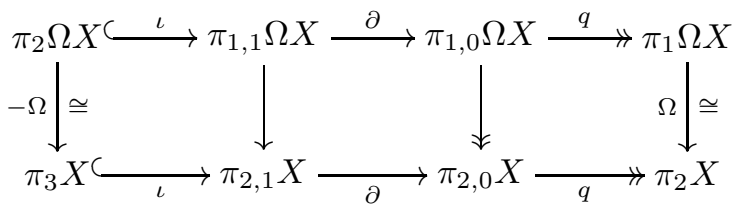

This diagram is analogue to (b) and shows that (c) is a weak equivalence.

Now for $n=0$ we define the functor $\pi_{0, *} \Omega X \rightarrow \phi_{1} \pi_{1, *} X$. On objects it is given by the inclusion $O b \pi_{0, *} \Omega X=\Omega X \subset\langle\Omega X\rangle=O b \phi_{1} \pi_{1, *} X$. Given any object $f \in \Omega X$ in $\pi_{0, *} \Omega X$ we consider the inclusion $\bar{f}: S^{1} \rightarrow S_{X}^{1}$ of the factor of the coproduct $S_{X}^{1}$ corresponding to $f$. Clearly the adjoint $a d\left(\bar{f}_{e v}\right): S^{0} \rightarrow \Omega X$ is the inclusion of the point $f \in \Omega X$. If $g \in \Omega X$ is another object then a morphism $H: f \rightarrow g$ in $\pi_{0, *} \Omega X$ is just a track $H: a d\left(\bar{f}_{e v}\right) \Rightarrow a d\left(\bar{g}_{e v}\right)$ in Top*. The functor sends the morphism $H$ to the element in $\pi_{1,0} X \ltimes \pi_{1,1} X$, which is $\pi_{1,0} X \times \pi_{1,1} X$ as a set, with $\left(\pi_{1} \bar{f}\right)(1)$ in the left coordinate and right coordinate given by the map

$$
S^{1} \stackrel{\mu}{\longrightarrow} S^{1} \vee S^{1} \stackrel{\nu \vee 1}{\longrightarrow} S^{1} \vee S^{1} \stackrel{(\bar{g}, \bar{f})}{\longrightarrow} S_{X}^{1}
$$


and the track

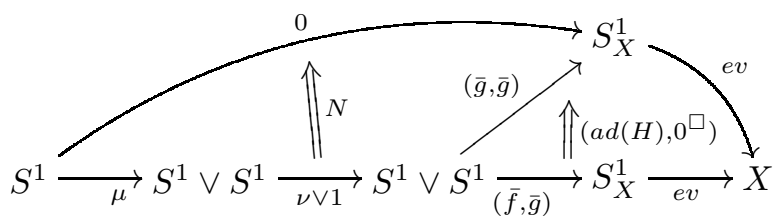

Here $N$ is a nil-track and $a d(H): \bar{f}_{e v} \Rightarrow \bar{g}_{e v}$ is the adjoint of the track $H$. We leave to the reader to check that $\pi_{0, *} \Omega X \rightarrow \phi_{1} \pi_{1, *} X$ is a well-defined functor. One can use again Proposition 5.1 to check that this functor is an equivalence.

The functors $\phi_{n}$ in (6.1) have left adjoints

$$
\operatorname{Ad}_{n}: \operatorname{group}(n-1) \longrightarrow \operatorname{group}(n)
$$

given by $\operatorname{Ad}_{n}=1_{\mathbf{A b}}$ for $n \geq 3$,

$$
\begin{aligned}
& \operatorname{Ad}_{2}: \mathbf{G r} \longrightarrow \mathbf{A b}, \text { the abelianization; } \\
& \operatorname{Ad}_{1}: \text { Set }^{*} \longrightarrow \mathbf{G r} \text {, taking free group. }
\end{aligned}
$$

These adjoints can be used to define for a pointed space $X$ the natural suspension morphisms

$$
\Sigma: \operatorname{Ad}_{n+1} \pi_{n} X \longrightarrow \pi_{n+1} \Sigma X
$$

as the adjoint of

$$
\pi_{n} X \stackrel{\pi_{n} a d(1)}{\longrightarrow} \pi_{n} \Omega \Sigma X \stackrel{\Omega}{\cong} \phi_{n+1} \pi_{n+1} \Sigma X .
$$

Here we use the map $a d(1): X \rightarrow \Omega \Sigma X$ which is adjoint to the identity in $\Sigma X$ and the natural isomorphism $\Omega$. Now we generalize the situation for secondary homotopy groups.

The functors $\phi_{n}$ in (6.2) have left adjoints,

$$
\operatorname{Ad}_{n}: \operatorname{cross}(n-1) \longrightarrow \operatorname{cross}(n)
$$

given by $\operatorname{Ad}_{n}=1_{\text {squad }}$ if $n \geq 4$,

$$
\begin{gathered}
\mathrm{Ad}_{3}: \operatorname{rquad} \longrightarrow \text { squad, } \\
\mathrm{Ad}_{2}: \text { cross } \longrightarrow \text { rquad, } \\
\mathrm{Ad}_{1}: \text { grd }^{*} \longrightarrow \text { cross }
\end{gathered}
$$

Lemma 6.9. The functors in [6.8) preserve 0 -free objects and weak equivalences between them.

Proof. For $\mathrm{Ad}_{1}$ the lemma follows from Lemma 6.12 below.

For $\mathrm{Ad}_{2}$ and $\mathrm{Ad}_{3}$ the lemma follows from the technical fact that the suspension functors between crossed and quadratic complexes described in Bau91 and Mur05, which are extensions of $\mathrm{Ad}_{2}$ and $\mathrm{Ad}_{3}$, are compatible with the homotopy relation in the category of totally free (i. e. cofibrant) crossed or quadratic complexes. In addition we use that 0 -free crossed or quadratic modules are exactly the truncations of totally free crossed or quadratic complexes.

The functor $\mathrm{Ad}_{3}$ is the stabilization in Bau91 IV.C.3. It is defined as follows. Given a reduced quadratic module

$$
(\omega, \partial)=\left(\otimes^{2} N_{a b} \stackrel{\omega}{\longrightarrow} M \stackrel{\partial}{\longrightarrow} N\right)
$$


the stabilized stable quadratic module

$$
\operatorname{Ad}_{3}(\omega, \partial)=\left(\otimes^{2} N_{a b} \stackrel{\omega_{\Sigma}}{\longrightarrow} M_{\Sigma} \stackrel{\partial_{\Sigma}}{\longrightarrow} N\right)
$$

is given by the group $M_{\Sigma}$ obtained by quotienting out in $M$ the relations

$$
\omega(a \otimes b+b \otimes a), \quad a, b \in N_{a b},
$$

and the homomorphisms $\omega_{\Sigma}$ and $\partial_{\Sigma}$ are induced by $\omega$ and $\partial$, respectively, in the obvious way.

The functor $\mathrm{Ad}_{2}$ in (6.8) is the suspension functor in [Mur05] 3.3. Given a crossed module $\partial: M \rightarrow N$ the reduced quadratic module

$$
\operatorname{Ad}_{2} \partial=\left(\otimes^{2} N_{a b} \stackrel{\omega}{\longrightarrow} M^{\tilde{\Sigma}} \stackrel{\delta}{\longrightarrow} N_{\text {nil }}\right)
$$

is given by the group $M^{\tilde{\Sigma}}$ which is a quotient of $M \times\left(\otimes^{2} N_{a b}\right)$ by the relations

$$
\left(-m+m^{n}, 0\right)=(0,\{\partial(m)\} \otimes\{n\})=(0,-\{n\} \otimes\{\partial(m)\}),
$$

for any $m \in M$ and $n \in N$; and the homomorphisms $\delta$ and $\omega$ are defined by the following formulas, $m \in M, n, n^{\prime} \in N$,

$$
\begin{aligned}
\delta\left(m,\{n\} \otimes\left\{n^{\prime}\right\}\right) & =\partial(m)+\left[n, n^{\prime}\right], \\
\omega\left(\{n\} \otimes\left\{n^{\prime}\right\}\right) & =\left(0,\{n\} \otimes\left\{n^{\prime}\right\}\right) .
\end{aligned}
$$

Finally we describe the functor $\operatorname{Ad}_{1}$. Let $\mathbf{G}$ be a groupoid with object pointed set $O b \mathbf{G}$ and morphism set $M o r \mathbf{G}$. The crossed module $\mathrm{Ad}_{1} \mathbf{G}$ is the quotient of the free crossed module, see Bau91, generated by the function

$$
\begin{gathered}
M o r \mathbf{G} \longrightarrow\langle O b \mathbf{G}\rangle \\
(h: U \rightarrow V) \mapsto-U+V
\end{gathered}
$$

by the relations $u=g+f$ for $u, f, g \in M$ or $\mathbf{G}$ with $u=f g$, the composition of $f$ and $g$ in $\mathbf{G}$. One readily checks that $\operatorname{Ad}_{1}$ is the adjoint of $\phi_{1}$.

The functor $h_{0}$ commutes with $\operatorname{Ad}_{n}$

$$
h_{0} \operatorname{Ad}_{n}=\operatorname{Ad}_{n} h_{0}, \quad n \geq 1 \text {. }
$$

This follows from the definition of $\operatorname{Ad}_{n}$ above for $n \geq 2$ and from Lemma 6.12 below in case $n=1$. For $h_{1}$ the corresponding commutativity law is not true in general, compare Lemma 6.12 below.

Theorem 6.11. There are natural morphisms in $\operatorname{cross}(n+1)$

$$
\Sigma: \operatorname{Ad}_{n+1} \pi_{n, *} X \longrightarrow \pi_{n+1, *} \Sigma X, n \geq 0,
$$

which induce the classical suspension homomorphism $\Sigma: \operatorname{Ad}_{n+1} \pi_{n} X \rightarrow \pi_{n+1} \Sigma X$ in $h_{0}$, and for $n \geq 3$ the homomorphism $-\Sigma: A_{n+2} \pi_{n+1} X \rightarrow \pi_{n+2} \Sigma X$ in $h_{1}$. Moreover, for $n \geq 3$ the morphism $\Sigma$ is a weak equivalence provided $X$ is $m$ connected and $n \leq 2 m-1$. It is also a weak equivalence for $n=2$ provided $X$ is simply connected, and for $n=1$ if $X$ is connected. Furthermore, $\Sigma$ is always a weak equivalence for $n=0$.

In the proof of this theorem we will use the following lemma.

Lemma 6.12. For any pointed groupoid $\mathbf{G}$ there are natural isomorphisms

(1) $h_{0} \operatorname{Ad}_{1} \mathbf{G}=\langle$ Iso $\mathbf{G}\rangle$,

(2) $h_{1} \operatorname{Ad}_{1} \mathbf{G}=\bigoplus_{x \in \operatorname{Iso}(\mathbf{G})}\left(\operatorname{Aut}_{\mathbf{G}}(x)\right)_{a b} \otimes R$. 
Here $R$ is the group ring of $\langle$ Iso $\mathbf{G}\rangle$.

Proof. The crossed module $\operatorname{Ad}_{1} \mathbf{G}$ defined above is the truncation

$$
N_{1} F B \mathbf{G} / d\left(N_{2} F B \mathbf{G}\right) \rightarrow N_{0} F B \mathbf{G}
$$

of the Moore complex $N_{*} F B \mathbf{G}$ of the Milnor construction $F B \mathbf{G}$ on the classifying space $B \mathbf{G}$ of the pointed groupoid $\mathbf{G}$, see Kan58 and GJ99 I.1.4 and V.6. To see this we have on the 0 -level

$$
N_{0} F B \mathbf{G}=\langle O b \mathbf{G}\rangle,
$$

and on the 1-level the set $M o r \mathbf{G}$ in $\operatorname{Ad}_{1} \mathbf{G}$ is mapped to $N_{1} F B \mathbf{G} / d\left(N_{2} F B \mathbf{G}\right)$ by sending $h: U \rightarrow V$ to the coset modulo $d\left(N_{2} F B \mathbf{G}\right)$ of the element

$$
-1_{U}+h \in N_{1} F B \mathbf{G} \subset F_{1} B \mathbf{G}=\langle M o r \mathbf{G}\rangle .
$$

This can be checked by computing $N_{1} F B \mathbf{G} / d\left(N_{2} F B \mathbf{G}\right)$ in terms of generators and relations. In order to carry out this computation one uses the Reidemeister-Schreier method, see [MKS66], which simplifies in this particular case since the simplicial identities hold in FB $\mathbf{G}$ and the boundaries and degeneracies in this simplicial group are homomorphisms between free groups on pointed sets induced by maps between the generating pointed sets.

By the previous observation the kernel and cokernel of $\operatorname{Ad}_{1} \mathbf{G}$ are the $\pi_{1}$ and $\pi_{2}$ of the suspension $\Sigma|B \mathbf{G}|$ of the geometric realization $|B \mathbf{G}|$ of the classifying space of $\mathbf{G}$, so the lemma follows from elementary facts from homotopy theory.

We also remark that given $x \in \operatorname{Iso}(\mathbf{G})$ and a representative $\tilde{x} \in O b \mathbf{G}$ of $x$ the group $\left(\operatorname{Aut}_{\mathbf{G}}(\tilde{x})\right)_{a b}$ does not depend on the choice of $\tilde{x}$, up to natural isomorphism, therefore we can denote it by $\left(\operatorname{Aut}_{\mathbf{G}}(x)\right)_{a b}$.

Proof of Theorem [6.11] Consider the morphism

$$
\pi_{n, *} X \stackrel{\pi_{n, *} \operatorname{ad}(1)}{\longrightarrow} \pi_{n, *} \Omega \Sigma X \stackrel{\Omega}{\longrightarrow} \phi_{n+1} \pi_{n+1, *} \Sigma X,
$$

where $\operatorname{ad}(1): X \rightarrow \Omega \Sigma X$ is the adjoint of the identity in $\Sigma X$ and $\Omega$ is given by Proposition 6.5 The morphism in the statement is the adjoint of this one. For $n \geq 3$ the range where this morphism is a weak equivalence follows from Proposition 5.1 and the classical suspension theorem for ordinary homotopy groups.

For $n=1$ the theorem follows from Proposition 8.5 below and [Mur05] 4.8. For $n=2$ we use Proposition 8.5 and Bau91] IV.C. For this we use that we are dealing with 0 -free objects and that $\operatorname{Ad}_{n}$ preserves weak equivalences between them, see Lemma 6.9

If $n=0$ we have $\operatorname{Iso}\left(\pi_{0, *} X\right)=\pi_{0} X$ and for any $x \in O b \pi_{0, *} X$, $\operatorname{Aut}_{\pi_{0, *} X}(x)=$ $\pi_{1}(X, x)$. By using elementary homotopy theory one can check that

$$
\pi_{1} \Sigma X \cong\left\langle\pi_{0} X\right\rangle
$$

and

$$
\pi_{2} \Sigma X \cong \bigoplus_{x \in \pi_{0} X}\left(\pi_{1}(X, x)\right)_{a b} \otimes \mathbb{Z}\left\langle\pi_{0} X\right\rangle .
$$

Now it is enough to notice that isomorphisms in Lemma 6.12 are compatible with the two isomorphisms above and Proposition 5.1 (in this last case up to sign -1 in kernel). 


\section{SECONDARY homotopy groups as TraCK FUnCTORS}

In Section 4.1 we have defined the secondary homotopy group functors $(n \geq 0)$

$$
\pi_{n, *}: \text { Top }^{*} \longrightarrow \operatorname{cross}(n) \text {. }
$$

As we recalled in Section 1 the category of pointed spaces is a track category, therefore it is reasonable to wonder whether $\pi_{n, *}$ is a track functor. This is known to be true if $n=0$. In this section we prove that ii is actually true for any $n \geq 0$.

We recall from Bau91 the definition of homotopy in the categories of crossed modules and reduced quadratic modules as follows.

Definition 7.1. Suppose that we have two crossed modules $\partial: M \rightarrow N, \partial^{\prime}: M^{\prime} \rightarrow$ $N^{\prime}$ and two morphisms $f, g: \partial \rightarrow \partial^{\prime}$ given by

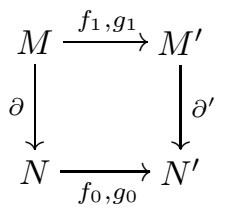

A track $\alpha: f \Rightarrow g$ is a function $\alpha: N \rightarrow M^{\prime}$ such that for any $x, y \in N$ and any $m \in M$ the following equalities hold:

(1) $\alpha(x+y)=\alpha(x)^{f_{0}(y)}+\alpha(y)$,

(2) $g_{0}(x)=f_{0}(x)+\partial^{\prime}(\alpha(x))$,

(3) $g_{1}(m)=f_{1}(m)+\alpha(\partial(m))$.

If we now have two reduced quadratic modules

$$
\begin{gathered}
\otimes^{2} N_{a b} \stackrel{\omega}{\longrightarrow} M \stackrel{\partial}{\longrightarrow} N, \\
\otimes^{2}\left(N^{\prime}\right)_{a b} \stackrel{\omega^{\prime}}{\longrightarrow} M^{\prime} \stackrel{\partial}{\longrightarrow} N^{\prime},
\end{gathered}
$$

and two morphisms $f, g:(\omega, \partial) \rightarrow\left(\omega^{\prime}, \partial^{\prime}\right)$

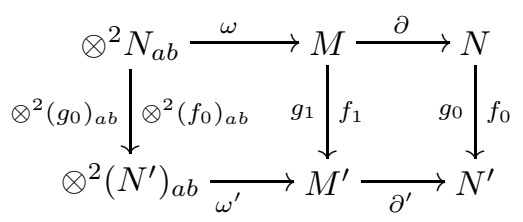

a track $\alpha: f \Rightarrow g$ is just a track $\alpha: \phi_{2} f \Rightarrow \phi_{2} g$ in the category of crossed modules. Here we use the forgetful functor $\phi_{2}$ in (6.3). More precisely, $\alpha: N \rightarrow M^{\prime}$ is a function such that for any $x, y \in N$ and $m \in M$ the following equations hold:

(1) $\alpha(x+y)=\alpha(x)+\alpha(y)+\omega^{\prime}\left(\left\{-f_{0}(x)+g_{0}(x)\right\} \otimes\left\{f_{0}(y)\right\}\right)$,

(2) $g_{0}(x)=f_{0}(x)+\partial^{\prime}(\alpha(x))$,

(3) $g_{1}(m)=f_{1}(m)+\alpha(\partial(m))$.

Tracks for stable quadratic module morphisms are the same as tracks for the corresponding reduced quadratic module morphisms. In particular the forgetful functors $\phi_{n}$ in (6.2) become automatically track functors which are full and faithful at the level of tracks for $n \geq 2$.

Tracks in the category grd* $^{*}$ of pointed groupoids are just natural transformations between functors. For $n=0$ the functor $\phi_{1}$ in (6.3) is also a track functor. More precisely, if $\partial: M \rightarrow N$ and $\partial^{\prime}: M^{\prime} \rightarrow N^{\prime}$ are crossed modules and $\alpha: N \rightarrow M^{\prime}$ is a track $\alpha: f \Rightarrow g$ between two morphisms $f, g: \partial \rightarrow \partial^{\prime}$ then the 
natural transformation $\phi_{1} \alpha: \phi_{1} f \Rightarrow \phi_{1} g$ between the pointed groupoid morphisms $\phi_{1} f, \phi_{1} g: \phi_{1} \partial \rightarrow \phi_{1} \partial^{\prime}$ is given by the morphisms $\left(f_{0}(n), \alpha(n)\right): f_{0}(n) \rightarrow g_{0}(n)$ in $\phi_{1} \partial^{\prime}$ which are natural in $n \in N$.

Proposition 7.2. The category $\operatorname{cross}(n)$ with tracks as in Definition 7.1 is a track category. Moreover, for all $n \geq 1$ the functor $\operatorname{Ad}_{n}$ in (6.8) is a track functor which is adjoint to $\phi_{n}$ in (6.3) as a track functor.

Proof. For $n=0$ it is well-known that $\operatorname{cross}(n)$ is a track category. We only need to carry out the proof of the first part of the statement for crossed modules since the track structure in rquad and squad is pulled back through the forgetful functors in (6.3).

In this proof $\partial_{i}: M_{i} \rightarrow N_{i}=\left\langle E_{i}\right\rangle$ will denote a crossed module for $i=0,1,2,3$.

Let $f, g, h: \partial_{1} \rightarrow \partial_{2}$ be crossed module morphisms and let $\alpha: f \Rightarrow g, \beta: g \Rightarrow h$ be vertically composable tracks. The vertical composition is defined by $(\beta \square \alpha)(x)=$ $\alpha(x)+\beta(x)$ for any $x \in M_{1}$. The inverse track $\alpha^{\boxminus}: g \Rightarrow f$ is defined by $\alpha^{\boxminus}(x)=$ $-\alpha(x)$ and the trivial track $0_{f}^{\square}: f \Rightarrow f$ is $0_{f}^{\square}(x)=0$.

Suppose that we have a diagram

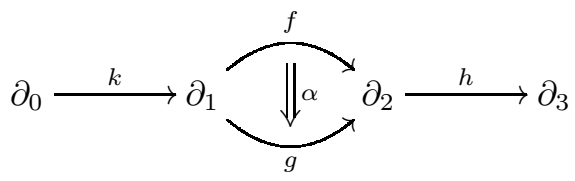

Then the two possible horizontal compositions $\alpha k: f k \Rightarrow g k, h \alpha: h f \Rightarrow g f$ are defined as $\alpha k=\alpha k_{0}: N_{0} \rightarrow M_{3}$ and $h \alpha=h_{1} \alpha: N_{1} \rightarrow M_{4}$.

Suppose now that we have a diagram

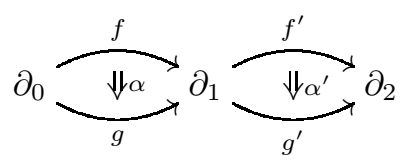

Let us check the equality

$$
\left(g^{\prime} \alpha\right) \square\left(\alpha^{\prime} f\right)=\left(\alpha^{\prime} g\right) \square\left(f^{\prime} \alpha\right) .
$$

Given $x \in M_{0}$ by using the equations defining crossed modules and tracks we get

$$
\begin{aligned}
\left(\left(g^{\prime} \alpha\right) \square\left(\alpha^{\prime} f\right)\right)(x) & =\alpha^{\prime} f_{0}(x)+g_{1}^{\prime} \alpha(x) \\
& =\alpha^{\prime} f_{0}(x)+f_{1}^{\prime} \alpha(x)+\alpha^{\prime} \partial_{1} \alpha(x) \\
& =\alpha^{\prime} f_{0}(x)+f_{1}^{\prime} \alpha(x)+\alpha^{\prime}\left(-f_{0}(x)+g_{0}(x)\right) \\
& =\alpha^{\prime} f_{0}(x)+f_{1}^{\prime} \alpha(x)+\alpha^{\prime}\left(-f_{0}(x)\right)^{f_{0}^{\prime} g_{0}(x)}+\alpha^{\prime}\left(g_{0}(x)\right) \\
& =\alpha^{\prime} f_{0}(x)+f_{1}^{\prime} \alpha(x)-\alpha^{\prime}\left(f_{0}(x)\right)^{f_{0}^{\prime}\left(-f_{0}(x)+g_{0}(x)\right)}+\alpha^{\prime}\left(g_{0}(x)\right) \\
& =\alpha^{\prime} f_{0}(x)+f_{1}^{\prime} \alpha(x)-\alpha^{\prime}\left(f_{0}(x)\right)^{f_{0}^{\prime} \partial_{1} \alpha(x)}+\alpha^{\prime}\left(g_{0}(x)\right) \\
& =\alpha^{\prime} f_{0}(x)+f_{1}^{\prime} \alpha(x)-\alpha^{\prime}\left(f_{0}(x)\right)^{\partial_{2} f_{1}^{\prime} \alpha(x)}+\alpha^{\prime}\left(g_{0}(x)\right) \\
& =\alpha^{\prime} f_{0}(x)-\alpha^{\prime}\left(f_{0}(x)\right)+f_{1}^{\prime} \alpha(x)+\alpha^{\prime}\left(g_{0}(x)\right) \\
& =f_{1}^{\prime} \alpha(x)+\alpha^{\prime}\left(g_{0}(x)\right) \\
& =\left(\left(\alpha^{\prime} g\right) \square\left(f^{\prime} \alpha\right)\right)(x) .
\end{aligned}
$$

Hence (a) holds and $\operatorname{cross}(1)$ is indeed a track category. 
Now we define the track functors $\operatorname{Ad}_{n}$ at the level of tracks. For $n \geq 4$ they are identity track functors. For $n=3$, given a track $\alpha: N \rightarrow M^{\prime}$ between two reduced quadratic module morphisms from $(\omega, \partial)$ to $\left(\omega^{\prime}, \partial^{\prime}\right)$ the track $\operatorname{Ad}_{3} \alpha: N \rightarrow M_{\Sigma}^{\prime}$ is the composition of $\alpha$ with the natural projection $M^{\prime} \rightarrow M_{\Sigma}^{\prime}$. For $n=2$, if $\alpha: N \rightarrow M^{\prime}$ is a track between two crossed module morphisms from $\partial$ to $\partial^{\prime}$ then $\operatorname{Ad}_{2} \alpha: N_{\text {nil }} \rightarrow$ $\left(M^{\prime}\right)^{\tilde{\Sigma}}$ is defined as $\left(\operatorname{Ad}_{2} \alpha\right)(n)=(\alpha(n), 0)$ for $n \in N_{n i l}$. Finally for $n=1$, if $\alpha: f \Rightarrow g$ is a natural transformation between two pointed groupoid morphisms $f, g: \mathbf{G} \rightarrow \mathbf{G}^{\prime}$ which is given by a collection of morphisms $\alpha(X): f(X) \rightarrow g(X)$ in $\mathbf{G}^{\prime}$ for $X \in O b \mathbf{G}$ then $\operatorname{Ad}_{1} \alpha:\langle O b \mathbf{G}\rangle \rightarrow\left(\operatorname{Ad}_{1} \mathbf{G}^{\prime}\right)_{1}$ is the unique track between crossed module morphisms satisfying $\left(\operatorname{Ad}_{1} \alpha\right)(X)=\alpha(X)$.

Theorem 7.3. The secondary homotopy groups are track functors $(n \geq 0)$

$$
\pi_{n, *}: \text { Top }^{*} \longrightarrow \operatorname{cross}(n) \text {. }
$$

In the proof of Theorem 7.3 we will use the following general construction.

Definition 7.4. Let $X$ be a pointed space. Given two maps $f, g: S^{1} \rightarrow \vee_{\Omega^{n} X} S^{1}$ and a track $H: f_{e v} \Rightarrow g_{e v}$ we define $r(H) \in \pi_{n, 1} X$ as follows. Let $\varepsilon: S^{1} \rightarrow S^{1} \vee S^{1}$ be a map with $\pi_{1} \varepsilon: \mathbb{Z} \rightarrow\langle a, b\rangle$ satisfying $\left(\pi_{1} \varepsilon\right)(1)=-a+b$, or just $\left(\pi_{1} \varepsilon\right)_{n i l}(1)=-a+b$ if $n \geq 2$, then $r(H)$ is represented by the map

$$
S^{1} \stackrel{\varepsilon}{\longrightarrow} S^{1} \vee S^{1} \stackrel{(f, g)}{\longrightarrow} \vee_{\Omega^{n} X} S^{1}
$$

and the track

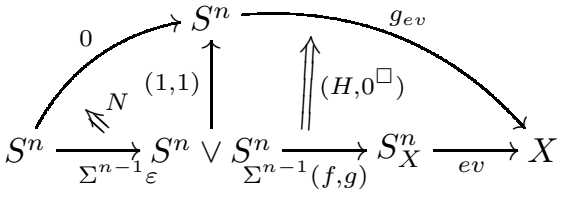

The $r$-construction may be regarded as a machine to generate homotopies in $\operatorname{cross}(n)$ between secondary homotopy groups. Some of the axioms of a homotopy in $\operatorname{cross}(n)$ are checked in the following lemma for the $r$-construction.

Lemma 7.5. Let $X$ be a pointed space. Given $f, g, h: S^{1} \rightarrow \vee_{\Omega^{n} X} S^{1}, H: f_{e v} \Rightarrow g_{e v}$ and $K: g_{e v} \Rightarrow h_{e v}$ the following formulas hold in $\pi n, 1 X$,

(1) $\partial r(H)=-\left(\pi_{1} f\right)(1)+\left(\pi_{1} g\right)(1)$ if $n=1$,

(2) $\partial r(H)=-\left(\pi_{1} f\right)_{n i l}(1)+\left(\pi_{1} g\right)_{n i l}(1)$ if $n \geq 2$,

(3) $r(K \square H)=r(H)+r(K)$.

Proof. Equations (1) and (2) are clear. The element $r(H)+r(K)$ is given by the following diagram

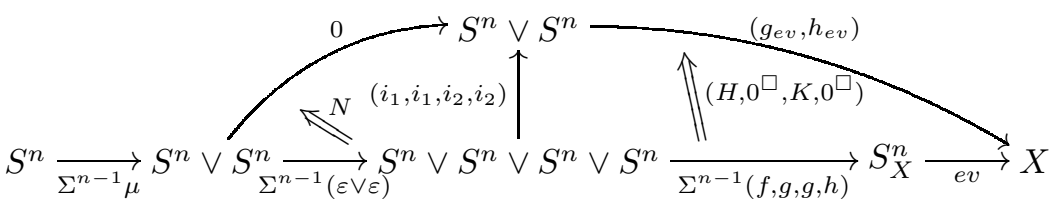


and this composite track coincides with the following ones
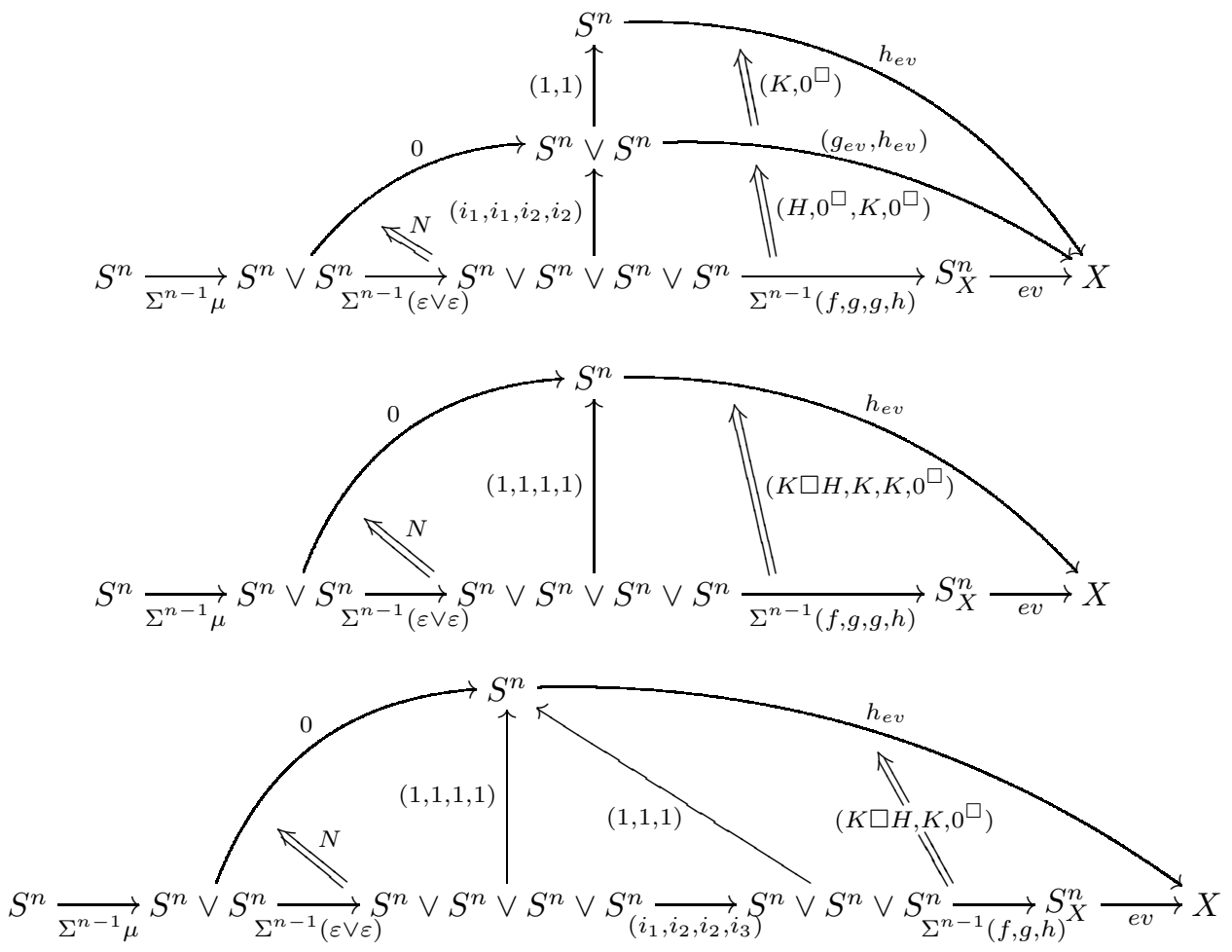

This composite track represents the same element in $\pi_{n, 1} X$ as

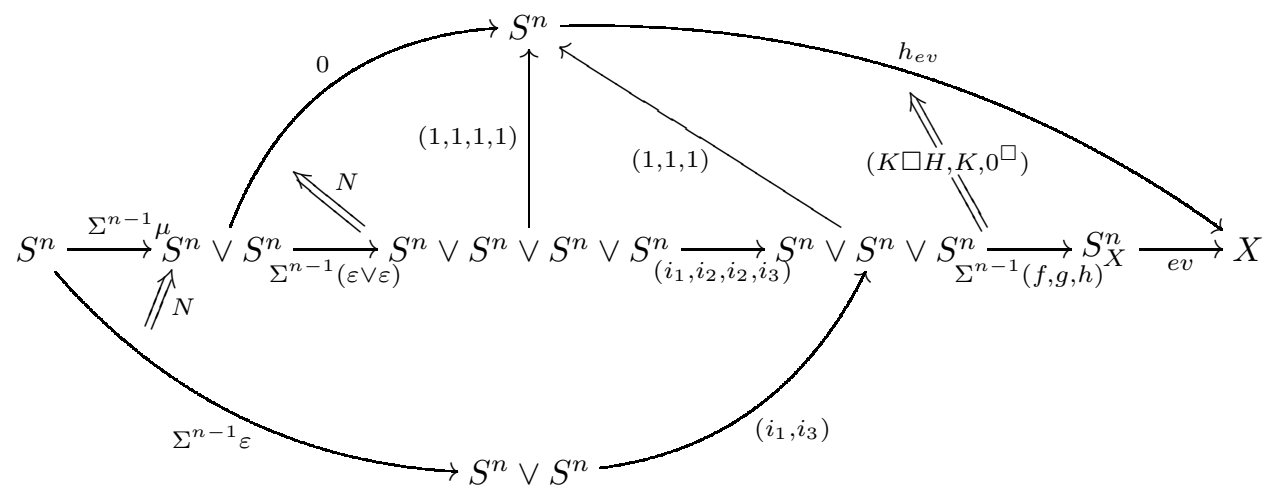

which is the same as

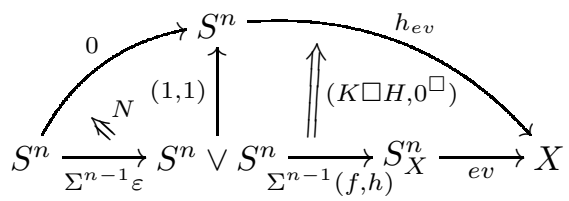

that is $r(K \square H)$, hence the chain of equalities proves (3).

In the next lemma we check the derivation property for the $r$-construction. 
Lemma 7.6. Let $X, Y$ be pointed spaces. Given $x, y: S^{1} \rightarrow \vee_{\Omega^{n} X} S^{1}, f, g: \vee_{\Omega^{n} X}$ $S^{1} \rightarrow \vee_{\Omega^{n} Y} S^{1}$, and $H: e v\left(\Sigma^{n-1} f\right) \Rightarrow e v\left(\Sigma^{n-1} g\right)$, we have the following equalities in $\pi_{n, 1} Y$ :

$$
\begin{aligned}
r\left(H\left(\Sigma^{n-1}(x, y)\right)\left(\Sigma^{n-1} \mu\right)\right)= & r\left(H\left(\Sigma^{n-1} x\right)\right)^{\pi_{1}(f y)(1)}+r\left(H\left(\Sigma^{n-1} y\right)\right) \text { if } n=1 \\
\text { and if } n \geq 2= & r\left(H\left(\Sigma^{n-1} x\right)\right)+r\left(H\left(\Sigma^{n-1} y\right)\right) \\
& +\omega\left(\left\{-\pi_{1}(f x)(1)+\pi_{1}(g x)(1)\right\},\left\{\pi_{1}(f y)(1)\right\}\right) .
\end{aligned}
$$

Proof. Suppose that $n=1$. Let $\varpi: S^{1} \rightarrow S^{1} \vee \stackrel{5}{\cdot} \vee S^{1}$ be a map with

$$
\pi_{1} \varpi: \mathbb{Z} \rightarrow\left\langle a_{1}, a_{2}, a_{3}, a_{4}, a_{5}\right\rangle: 1 \mapsto-a_{1}-a_{2}+a_{3}+a_{1}-a_{4}+a_{5} .
$$

The element $r\left(H\left(\Sigma^{n-1} x\right)\right)^{\pi_{1}(f y)(1)}+r\left(H\left(\Sigma^{n-1} y\right)\right)$ is represented by the diagram

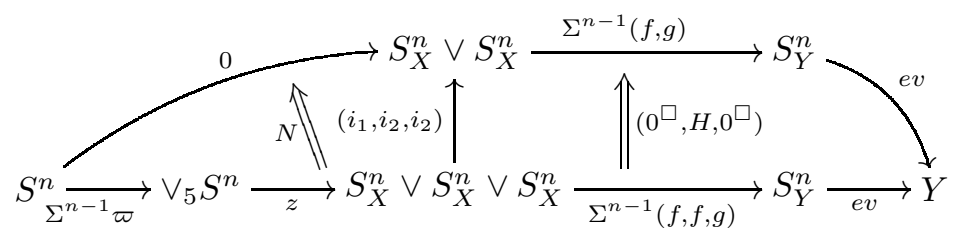

where $z=\Sigma^{n-1}\left(i_{1} y, i_{2} x, i_{3} x, i_{2} y, i_{3} y\right)$. This composite track coincides with

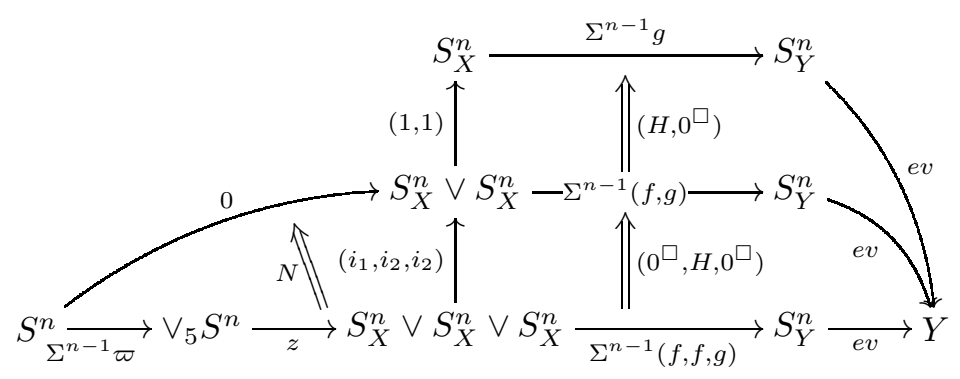

which is the same as

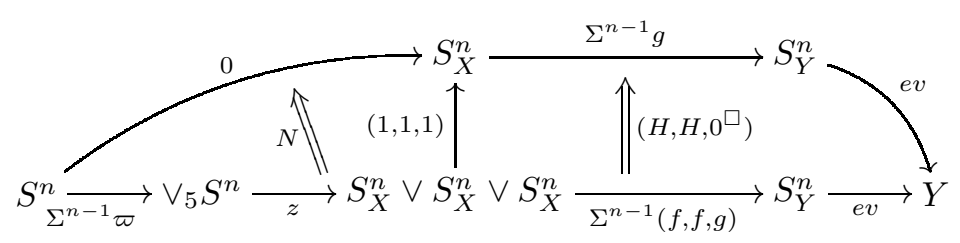

This one is the same as

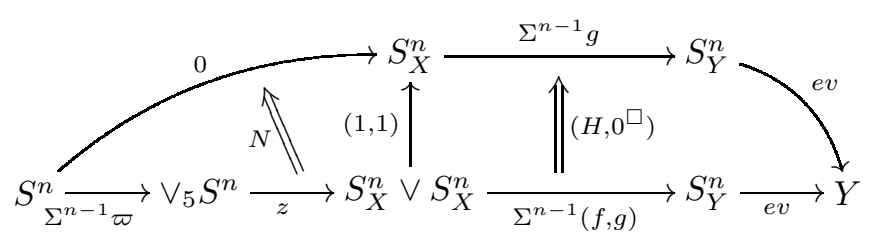


This composite track represents the same element in $\pi_{1,1} Y$ as

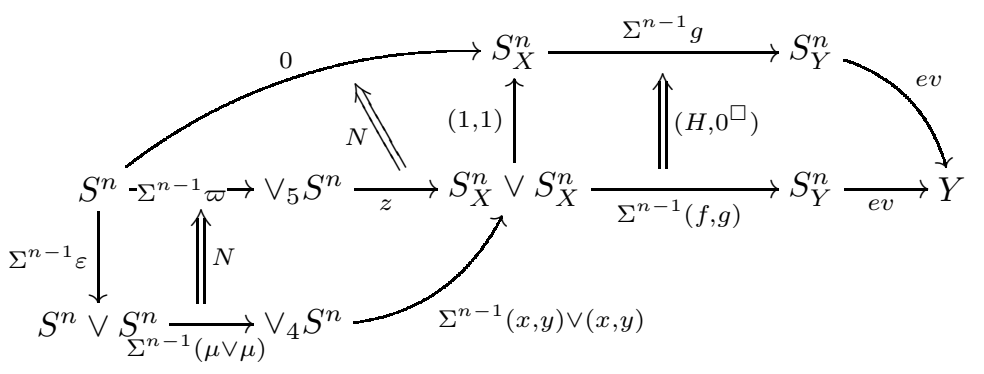

and this is $r\left(H\left(\Sigma^{n-1}(x, y)\right)\left(\Sigma^{n-1} \mu\right)\right)$.

Suppose now that $n \geq 2$. By using Theorem 3.6 and the claim $\left(^{*}\right)$ in the proof of Proposition 4.9 the element

$$
r\left(H\left(\Sigma^{n-1} x\right)\right)+r\left(H\left(\Sigma^{n-1} y\right)\right)+\omega\left(\left\{-\pi_{1}(f x)(1)+\pi_{1}(g x)(1)\right\},\left\{\pi_{1}(f y)(1)\right\}\right)
$$

is represented by the diagram

(a)

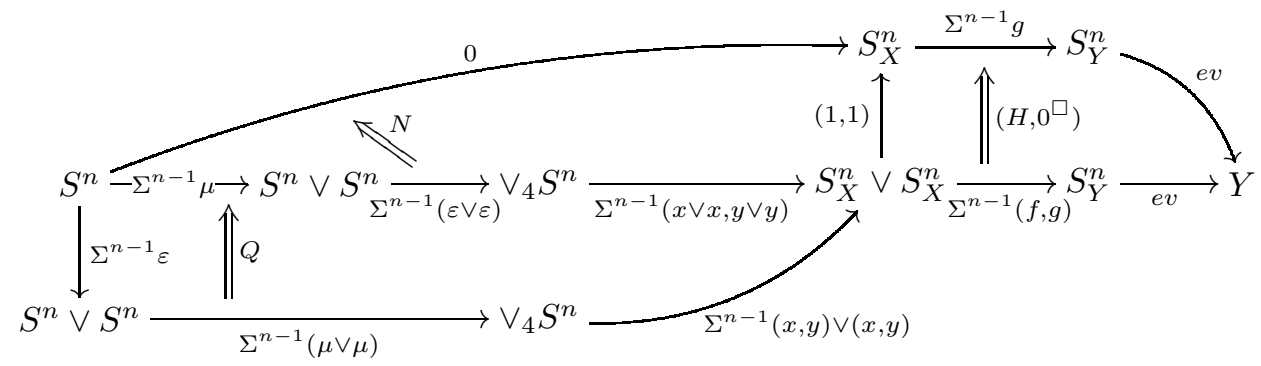

where $Q$ is the unique nil-track with Hopf invariant

$$
-\left(-i_{1}\left(\pi_{1}(x)\right)_{a b}(1)+i_{2}\left(\pi_{1}(x)\right)_{a b}(1)\right) \otimes\left(i_{1}\left(\pi_{1}(y)\right)_{a b}(1)\right) \in \otimes_{n}^{2}\left(\mathbb{Z}\left[\Omega^{n} X\right] \oplus \mathbb{Z}\left[\Omega^{n} X\right]\right),
$$

for $i_{1}, i_{2}: \mathbb{Z}\left[\Omega^{n} X\right] \rightarrow \mathbb{Z}\left[\Omega^{n} X\right] \oplus \mathbb{Z}\left[\Omega^{n} X\right]$ the inclusion of the factors of the direct sum. By Theorem 3.6 the track $(1,1) Q$ is a nil-track, hence diagram (a) also represents the element $r\left(H\left(\Sigma^{n-1}(x, y)\right)\left(\Sigma^{n-1} \mu\right)\right)$, and we are done.

Now we are ready to prove the main result of this section.

Proof of Theorem 7.3. This is known for $n=0$. Suppose now that $n \geq 1$. Let $f, g: X \rightarrow Y$ be two maps and $f_{*}, g_{*}: \pi_{n, *} X \rightarrow \pi_{n, *} Y$ the induced morphisms in $\operatorname{cross}(n)$. Moreover, let $H: f \Rightarrow g$ be a track. We define a track $\pi_{n, *} H=$ $H_{*}: f_{*} \Rightarrow g_{*}$ in the following way. The function $H_{*}: \pi_{n, 0} X \rightarrow \pi_{n, 1} Y$ sends an element $x \in \pi_{n, 0} X$ represented by a map $\tilde{x}: S^{1} \rightarrow \vee_{\Omega^{n} X} S^{1}$ with $\left(\pi_{1} \tilde{x}\right)(1)=x$ if $n=1$ and $\left(\pi_{1} \tilde{x}\right)_{n i l}(1)=x$ if $n \geq 2$ to the element in $\pi_{n, 1} Y$ represented by the map (a) and the track (b) below.

Let $\varepsilon: S^{1} \rightarrow S^{1} \vee S^{1}$ be a map with $\left(\pi_{1} \varepsilon\right)(1)=-a+b \in\langle a, b\rangle$. The map (a) is defined by

$$
S^{1} \stackrel{\varepsilon}{\longrightarrow} S^{1} \vee S^{1} \stackrel{\tilde{x} \vee \tilde{x}}{\longrightarrow}\left(\vee_{\Omega^{n} X} S^{1}\right) \vee\left(\vee_{\Omega^{n} X} S^{1}\right) \stackrel{\Sigma \Omega^{n}(f, g)}{\longrightarrow} \vee_{\Omega^{n} Y} S^{1} .
$$


The track (b) is given by

(b)

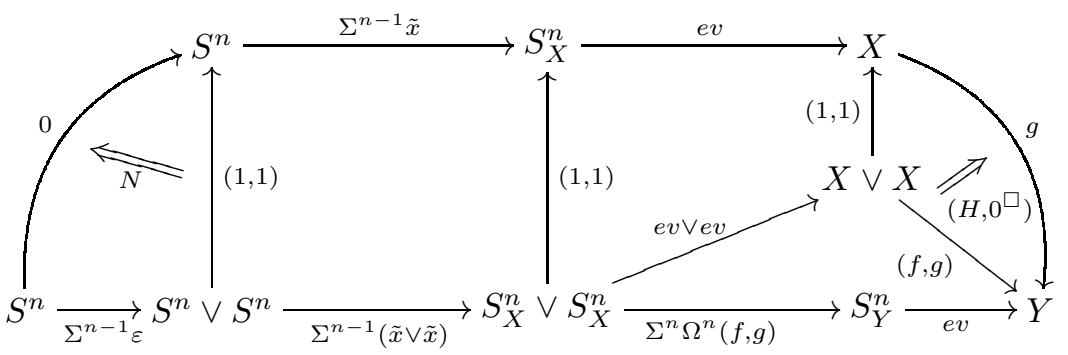

Here $N$ is a nil-track. With the terminology introduced in Definition 7.4 we have $H_{*}(x)=r\left(H e v\left(\Sigma^{n-1} \tilde{x}\right)\right)$ for the track $H e v\left(\Sigma^{n-1} \tilde{x}\right): \operatorname{ev}\left(\sum^{n} \Omega^{n} f\right)\left(\sum^{n-1} \tilde{x}\right)=$ $f \operatorname{ev}\left(\Sigma^{n-1} \tilde{x}\right) \Rightarrow g \operatorname{ev}\left(\Sigma^{n-1} \tilde{x}=\operatorname{ev}\left(\Sigma^{n} \Omega^{n} g\right)\left(\Sigma^{n-1} \tilde{x}\right.\right.$.

The proof of equation (1) in the definition of tracks in $\operatorname{cross}(n)$ follows from Lemma 7.6. Equation (2) follows from Lemma 7.5(1) or (2). Equation (3) follows from the fact that given $[k, K] \in \pi_{n, 1} X$ the following composite tracks coincide.
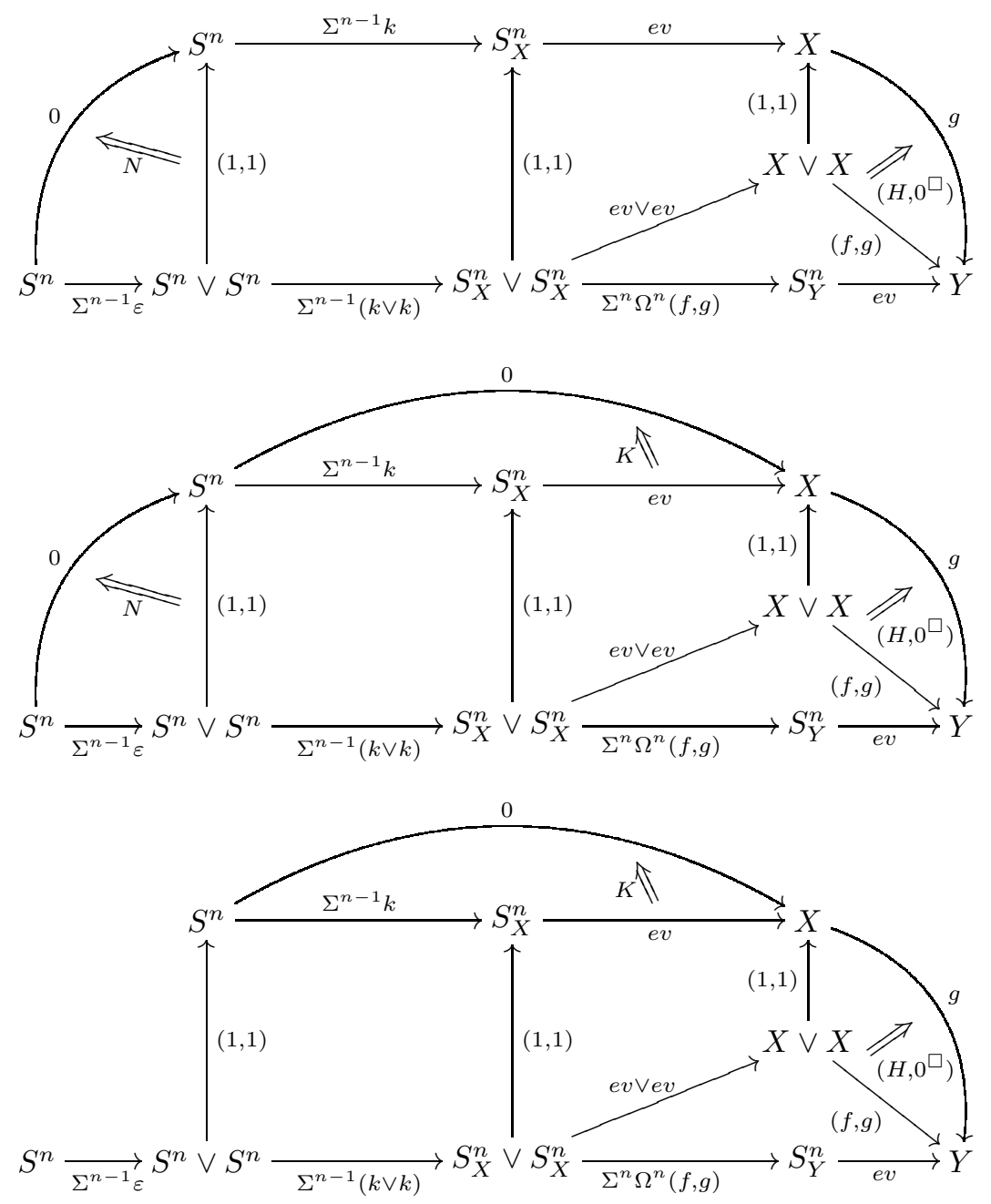


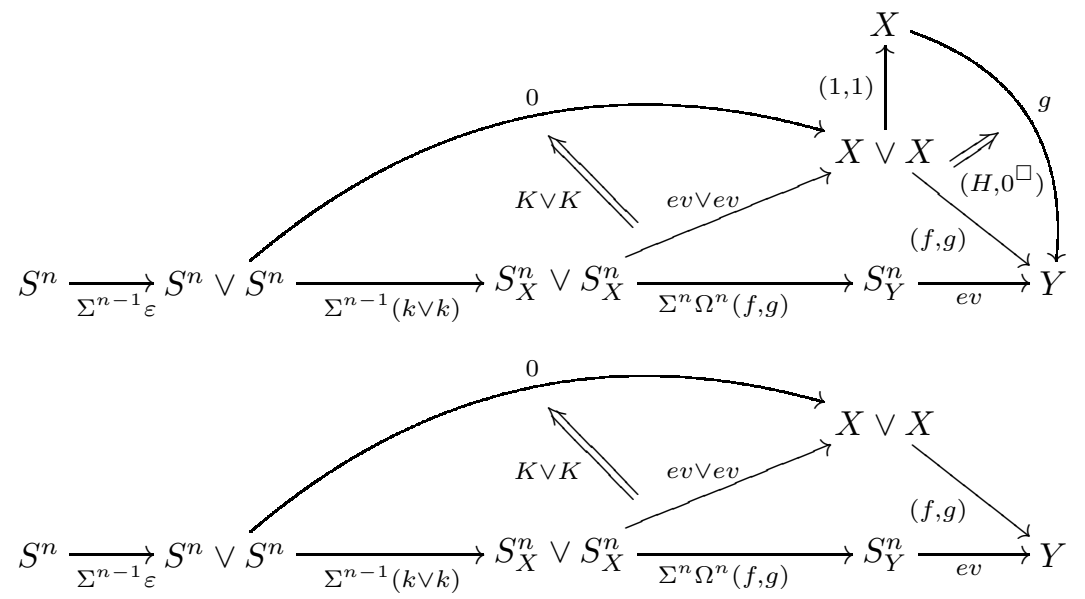

The vertical composition of tracks $f \stackrel{H}{\Rightarrow} g \stackrel{K}{\Rightarrow} h$ is preserved by Lemma 7.5 (3). The proof of the fact that $\pi_{n, *}$ preserves horizontal composition is straightforward and it is left to the reader.

Proposition 7.7. The inclusion $\operatorname{cross}_{f}(n) \subset \operatorname{cross}(n)$ of the full subcategory of 0 -free objects induces an equivalence of categories $(n \geq 0)$

$$
\operatorname{cross}_{f}(n) / \simeq \stackrel{\sim}{\longrightarrow} \text { Ho } \operatorname{cross}(n),
$$

where the homotopy category Ho is obtained by inverting weak equivalences.

Proof. For $n=0$ this result is well-known. For $n=1$ this is a consequence of the fact that cross has a model category structure where 0 -free objects are the cofibrant objects, see [GM97, and the homotopy relation derived from the cylinders on cofibrant objects is given by the tracks defined above. In a similar way one obtains the result for $n \geq 2$.

\section{8. $k$-INVARIANTS}

Let $K(G, n)$ be the Eilenberg-MacLane space with $\pi_{n} K(G, n)=G$. Following Eilenberg-MacLane's notation we write $H^{m}(G, n, A)$ for the $m$-dimensional cohomology of the space $K(G, n)$ with coefficients in the abelian group $A$. Here we allow $A$ to be a $G$-module in case $n=1$. In this case $H^{m}(G, 1, A)=H^{m}(G, A)$ is the ordinary cohomology (with local coefficients) of the group $G$.

For any connected $C W$-complex $X$ we write

$$
k_{n}(X) \in H^{n+2}\left(\pi_{n} X, n, \pi_{n+1} X\right)
$$

for the first $k$-invariant of the $(n-1)$-connected cover $X\langle n\rangle$. Recall that $X\langle n\rangle$ is the homotopy fiber of the canonical map from $X$ to its $(n-1)$-type, $X \rightarrow P_{n-1} X$, where $P_{n-1} X$ is a Postnikov section of $X$.

If $n=1$ then $k_{1}(X)$ is the usual first $k$-invariant of a connected $C W$-complex $X$, represented by the crossed module

$$
\partial: \pi_{2}\left(X, X^{1}\right) \longrightarrow \pi_{1} X^{1}
$$

determined by the skeletal filtration of $X$, see MW50. Otherwise, if $n \geq 2$

$$
H^{n+2}\left(\pi_{n} X, n, \pi_{n+1} X\right)=\operatorname{Hom}\left(\Gamma_{n} \pi_{n} X, \pi_{n+1} X\right)
$$


and $k_{n}(X): \Gamma_{n} \pi_{n} X \rightarrow \pi_{n+1} X$ is induced by the function $\eta^{*}: \pi_{n} X \rightarrow \pi_{n+1} X$ which sends the homotopy class of $\alpha: S^{n} \rightarrow X$ to the homotopy class of $\alpha\left(\Sigma^{n-2} \eta\right): S^{n+1} \rightarrow$ $X$, where $\eta: S^{3} \rightarrow S^{2}$ is the Hopf map. Compare notation in (2.2).

The first secondary homotopy group $\pi_{1, *} X$ is a crossed module, see Proposition 4.6. By Proposition 5.1 and MW50 this crossed module represents an element

$$
k\left(\pi_{1, *} X\right) \in H^{3}\left(\pi_{1} X, \pi_{2} X\right) .
$$

In general, any crossed module $\partial$ defines a cohomology class $k(\partial) \in H^{3}\left(h_{0} \partial, h_{1} \partial\right)$, see [MW50].

For $n \geq 2$ the $n$-dimensional secondary homotopy group of $X$ defines a homomorphism

$$
k\left(\pi_{n, *} X\right): \Gamma_{n} \pi_{n} X \longrightarrow \pi_{n+1} X,
$$

as follows. Let $k\left(\pi_{n, *} X\right)$ be the unique homomorphism fitting into the following commutative diagram

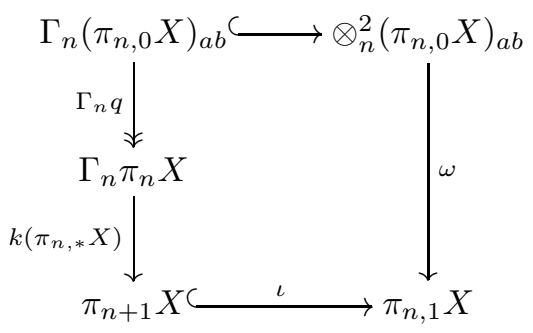

Here the upper horizontal arrow is the injection in (2.2), and $\iota$ and $q$ appear in Proposition 5.1

In general any 0 -free reduced quadratic module $(\omega, \partial)$ defines a homomorphism

$$
k(\omega, \partial): \Gamma h_{0}(\partial, \omega) \longrightarrow h_{1}(\omega, \partial),
$$

as in (8.1) and any 0 -free stable quadratic module $(\omega, \partial)$ defines accordingly a homomorphism

$$
k(\omega, \partial): h_{0}(\omega, \partial) \otimes \mathbb{Z} / 2 \longrightarrow h_{1}(\omega, \partial) .
$$

Theorem 8.2. For any connected $C W$-complex $X$ and any $n \geq 1$ the equality $k_{n}(X)=k\left(\pi_{n, *} X\right)$ holds.

Proof. We can suppose without loss of generality that the 1-skeleton $X^{1}=\vee_{E} S^{1}$ is just a one-point union of 1 -spheres. One can easily check that $\pi_{1, *}(X, E)$ in Proposition 4.15 coincides with $\partial: \pi_{2}\left(X, X^{1}\right) \rightarrow \pi_{1} X^{1}$, hence the theorem follows for $n=1$.

We now prove the theorem for $n \geq 2$. Suppose that we have $x \in \pi_{n, 0} X$ and we choose $\tilde{x}: S^{1} \rightarrow \vee_{\Omega^{n} X} S^{1}$ with $\left(\pi_{1} \tilde{x}\right)_{n i l}(1)=x$. Then $\omega(\{x\} \otimes\{x\}) \in \pi_{n, 1} X$ is represented by

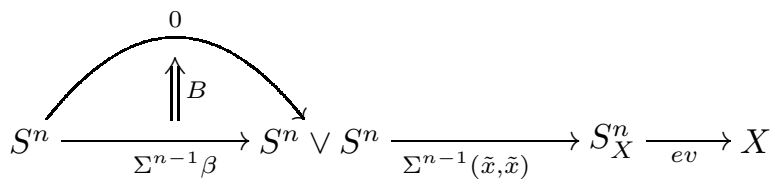


This is the same as

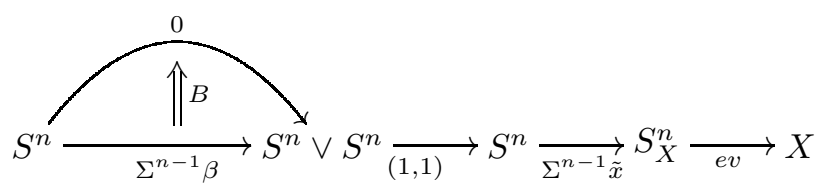

By Theorem 3.6

$$
\operatorname{Hopf}((1,1) B)=-1 \in \otimes_{n}^{2} \mathbb{Z}=\left\{\begin{array}{cc}
\mathbb{Z}, & \text { if } n=2 ; \\
\mathbb{Z} / 2, & \text { if } n \geq 3 .
\end{array}\right.
$$

Moreover, $\left(\pi_{1}(1,1) \beta\right)_{n i l}=0$, therefore by using the definition of $\iota$ in Proposition 5.1. Theorem [3.6] Remark 3.4 and the characterization of $\eta: S^{3} \rightarrow S^{2}$ up to homotopy as the unique map with Hopf invariant 1 we get that $\omega(\{x\} \otimes\{x\})=\iota\left(q(x)\left(\Sigma^{n-2} \eta\right)\right)$, hence we are done.

Let types ${ }_{n}^{1}$ be the category of pointed $(n-1)$-connected $C W$-complexes $X$ with $\pi_{m}\left(X, x_{0}\right)=0$ for all $m \geq n+2$ and all $x_{0} \in X$.

Proposition 8.3. The functor $\pi_{n, *}: \operatorname{types}_{n}^{1} \rightarrow \operatorname{cross}(n)$ induces an equivalence of categories $(n \geq 0)$

$$
\pi_{n, *}: \operatorname{Hotypes}_{n}^{1} \stackrel{\sim}{\longrightarrow} \operatorname{Ho} \operatorname{cross}(n),
$$

where the homotopy category Ho is obtained by localizing with respect to weak equivalences.

For the proof of Proposition 8.3 we recall the following functors.

Let $\mathbf{C W}_{n}$ be the category of $C W$-complexes $X$ with trivial $(n-1)$-skeleton $X^{n-1}=*$ and cellular maps. There is a "cellular" functor

$$
P_{n+1} \sigma: \mathbf{C W}_{n} / \simeq \longrightarrow \operatorname{cross}(n) / \simeq .
$$

If $n=1$ this functor sends a $C W$-complex $X$ to the crossed module

$$
\partial: \pi_{2}\left(X, X^{1}\right) \rightarrow \pi_{1} X^{1}
$$

given by the boundary operator in the long exact sequence of homotopy groups, see [Mac49] and [MW50. If $n \geq 2$ the the reduced (stable if $n \geq 3$ ) quadratic module $P_{n} \sigma(X)$ is the truncation of the totally free quadratic complex $\sigma(X)$ defined in Bau91 IV.C,

$$
\otimes^{2} C_{n}(X) \stackrel{\omega}{\longrightarrow} \sigma_{n+1}(X) / d\left(\sigma_{n+2}(X)\right) \stackrel{\partial}{\longrightarrow} \sigma_{n}(X),
$$

compare Bau91 IV.10.4 and Mur05 4.

Proposition 8.5. The functor $P_{n+1} \sigma$ in 8.4 is naturally isomorphic to

$$
\pi_{n, *}: \mathbf{C W}_{n} / \simeq \rightarrow \operatorname{cross}_{f}(n) / \simeq
$$

for all $n \geq 1$.

Proof. If $X$ is $(n-1)$-reduced then $X^{n}=\vee_{E} S^{n}$ for some pointed set $E$. The inclusion of spheres in the wedge $X^{n} \subset X$ determines a pointed inclusion $E \subset \Omega^{n} X$. One can easily check that $P_{n+1} \sigma(X)$ is isomorphic to $\pi_{n, *}(X, E)$ in Proposition 4.15 , Now the natural isomorphism in the statement is given by the weak equivalence $P_{n+1} \sigma(X) \cong \pi_{n, *}(X, E) \stackrel{\sim}{\rightarrow} \pi_{n, *} X$ in Proposition 4.15 Compare Proposition 7.7 
Proof of 8.3. For $n=0$ this is a well-known result. For $n \geq 1$ this follows from Proposition 8.5 and the fact that $P_{n+1} \sigma$ in 8.4 does induce an equivalence of categories $P_{n+1} \sigma$ : Hotypes ${ }_{n}^{1} \rightarrow \operatorname{Hocross}(n)$. This is shown in Bau91] III.8.2 for $n=1$. For $n=2$ the proof follows as in the case $n=1$, this case is considered even in the non-simply connected case in Bau91 IV.10.1. The case $n \geq 3$ can be easily proved along the lines of the $n=1$ and $n=2$ cases, i. e. by using [Bau91] III.8.5, III.8.8 and IV.C.14.

Remark 8.6. In the literature there are further algebraic categories equivalent to Ho types $_{n}^{1}$. In particular, see for $n=1$ Tak05, for $n=2$ see [CC96], and for $n \geq 3$ BCC93. These algebraic models, by Proposition 8.3, can also be deduced from objects in $\operatorname{cross}(n)$. The objects in $\operatorname{cross}(n)$ seem to be the "smallest possible" algebraic objects representing the category Hotypes ${ }_{n}^{1}$. In addition the definition of these other algebraic models is not topological, but simplicial. The difference between our models and the other ones is similar to the difference between classical homotopy groups as homotopy classes of maps $S^{n} \rightarrow X$ and as the homology of the Moore complex of the Kan loop group of the singular simplicial set on $X$.

\section{REFERENCES}

[Bau] H.-J. Baues, The algebra of secondary cohomology operations, To appear in Progress in Math. Birkhäuser.

[Bau91] Combinatorial Homotopy and 4-Dimensional Complexes, Walter de Gruyter, Berlin, 1991.

[BCC93] M. Bullejos, P. Carrasco, and A. M. Cegarra, Cohomology with coefficients in symmetric cat-groups. An extension of Eilenberg-Mac Lane's classification theorem, Math. Proc. Cambridge Philos. Soc. 114 (1993), no. 1, 163-189.

[BJP05] H.-J. Baues, M. Jibladze, and T. Pirashvili, Quadratic algebra of square groups, Preprint, 2005.

[CC96] P. Carrasco and A. M. Cegarra, (Braided) tensor structures on homotopy groupoids and nerves of (braided) categorical groups, Comm. Algebra 24 (1996), no. 13, 3995-4058.

[Con84] D. Conduché, Modules croisés généralisés de longueur 2, J. Pure Appl. Algebra 34 (1984), no. 2-3, 155-178.

[GJ99] P. J. Goerss and J. F. Jardine, Simplicial Homotopy Theory, Progress in Mathematics, no. 174, Birkhäuser Verlag, Basel, 1999.

[GM97] A. R. Garzón and J. G. Miranda, Homotopy theory for (braided) CAT-groups, Cahiers Topologie et Géom. Différentielle Catégoriques 38 (1997), no. 2, 99-139.

[Kan58] D. M. Kan, A combinatorial definition of homotopy groups, The Annals of Mathematics 67 (1958), no. 2, 282-312.

[Mac49] S. MacLane, Cohomology theory in abstract groups III. operator homomorphisms of kernels., Ann. of Math. (2) 50 (1949), 736-761.

[MKS66] W. Magnus, A. Karras, and D. Solitar, Combinatorial Group Theory, Interscience, New York, 1966.

[Mur05] F. Muro, Suspensions of crossed and quadratic complexes, co-h-structures and applications, Trans. Amer. Math. Soc. 357 (2005), no. 9, 3623-3653.

[MW50] S. MacLane and J. H. C. Whitehead, On the 3-type of a complex, Proc. Nat. Acad. Sci. 36 (1950), 41-48.

[Tak05] M. Takuo, The homotopy category of certain topological monoidal categories, Proceedings of the International Conference on Homotopy Theory and Related Topics (Seoul) (M. H. Woo, ed.), Korea University, 2005, pp. 31-38.

[Tod62] H. Toda, Composition methods in homotopy groups of spheres, Annals of Mathematics Studies, No. 49, Princeton University Press, Princeton, N.J., 1962.

[Whi41] J. H. C. Whitehead, On adding relations to homotopy groups, Ann. of Math. (2) 42 (1941), 409-428.

[Whi49] Combinatorial homotopy II, Bull. Amer. Math. Soc. 55 (1949), 453-496.

[Whi50] _ A certain exact sequence, Ann. Math. 52 (1950), 51-110. 
Max-Planck-Institut für Mathematik, Vivatsgasse 7, 53111 Bonn, Germany

E-mail address: baues@mpim-bonn.mpg.de, muro@mpim-bonn.mpg.de 